\title{
Semi-empirical metallicity calibrations based on ultraviolet emission lines of type-2 AGNs
}

\author{
O. L. Dors, ${ }^{1 \star}$ A. F. Monteiro, ${ }^{\star}{ }^{\star}$ M. V. Cardaci, ${ }^{2,3}$ G. F. Hägele ${ }^{2,3}$ and A. C. Krabbe ${ }^{1}$ \\ ${ }^{1}$ Universidade do Vale do Paraíba, Av. Shishima Hifumi, 2911, Cep 12244-000, São José dos Campos, SP, Brazil \\ ${ }^{2}$ Instituto de Astrofísica de La Plata (CONICET-UNLP), Observatorio Astronómico de La Plata, Avenida Centenario (Paseo del Bosque) S/N, \\ B1900FWA La Plata, Argentina \\ ${ }^{3}$ Facultad de Ciencias Astronómicas y Geofísicas, Universidad Nacional de La Plata, Paseo del Bosque s/n, 1900 La Plata, Argentina
}

Accepted 2019 May 1. Received 2019 April 30; in original form 2018 December 27

\begin{abstract}
We derived two semi-empirical calibrations between the metallicity of the narrow-line region (NLR) of type-2 active galactic nuclei and the rest frame of the $\mathrm{N} V \lambda 1240 / \mathrm{He}$ II $\lambda 1640$, $\mathrm{C} 43=\log [(\mathrm{C}$ IV $\lambda 1549+\mathrm{C}$ III $] \lambda 1909) / \mathrm{He}$ II $\lambda 1640]$, and C III $] \lambda 1909 / \mathrm{C}$ IV $\lambda 1549$ emissionline intensity ratios. A metallicity-independent calibration between the ionization parameter and the C III] $\lambda 1909 / C$ IV $\lambda 1549$ emission-lines ratio was also derived. These calibrations were obtained comparing ratios of measured UV emission-line intensities, compiled from the literature, for a sample of 77 objects (redshift $0<z<3.8$ ) with those predicted by a grid of photoionization models built with the CLOUDY code. Using the derived calibrations, it was possible to show that the metallicity estimations for NLRs are lower by a factor of about 2-3 than those for broad-line regions. Besides, we confirmed the recent result of the existence of a relation between the stellar mass of the host galaxy and its NLR metallicity. We also derived an $M-Z$ relation for the objects in our sample at $1.6<z<3.8$. This relation seems to follow the same trend as the ones estimated for star-forming galaxies of similar high redshifts but for higher masses.
\end{abstract}

Key words: galaxies: abundances-galaxies: active-galaxies: evolution-galaxies: formation-galaxies: ISM-galaxies: nuclei.

\section{INTRODUCTION}

Active galaxy nuclei (AGNs) present prominent metal emission lines in their spectra, easily measured in a wide range of wavelengths even when the objects are located at very high redshift $(z>$ 5 ). The intensity of these lines can be used to estimate the gas phase metallicity, becoming AGNs essential in studies of chemical evolution of galaxies as well as of the Universe.

In general, it is assumed that fiducial metallicity $(Z)$ estimations of emitter line objects (e.g. AGNs, H II regions, and planetary nebulae) are those obtained through the $T_{\mathrm{e}}$-method (Osterbrock \& Ferland 2006), which, basically, consists of using collisionally excited forbidden emission lines to estimate the electron temperature and the abundance of a given element in relation to the hydrogen abundance, usually O/H. However, Dors et al. (2015) showed that oxygen abundances, estimated from the $T_{\mathrm{e}}$-method and from narrow emission lines of a sample of Seyfert 2 AGNs, are underestimated by up to $\sim 2$ dex (with averaged value of $\sim 0.8 \mathrm{dex}$ ) in relation to the expected values from the extrapolation of radial abundance

^E-mail: olidors@univap.br (OLD); adrianofcom@yahoo.com.br (AFM) gradients. These authors also showed that estimations of metallicity based on theoretical calibrations of strong optical emission lines seem to be reliable for AGNs.

Most of the metallicity calibrations for AGNs (Storchi-Bergmann et al. 1998; Castro et al. 2017) for H II regions (e.g. Pagel et al. 1979; Edmunds \& Pagel 1984; Pilyugin 2000, 2001; Kewley \& Dopita 2002; Dors \& Copetti 2005; Stasińska 2006; Maiolino et al. 2008; Berg, Skillman \& Marble 2011; Pérez-Montero 2014; Brown, Martini \& Andrews 2016; Pilyugin \& Grebel 2016) and even for diffuse ionized gas (Kumari et al. 2019) are based on strong optical emission lines (e.g. [O II] $\lambda 3727, \mathrm{H} \beta$, [O III] $\lambda 5007$, [N II] $\lambda 6584, \mathrm{H} \alpha)$. These calibrations, together with the large amount of optical spectroscopic data obtained by several surveys, such as the Calar Alto Legacy Integral Field Area (CALIFA) survey (Sánchez et al. 2012) and the Sloan Digital Sky Survey (SDSS; York et al. 2000), have revolutionized the extragalactic astronomy. However, optical emission lines shift out of the $K$ band atmospheric window for objects at high redshifts $(z>3.5)$, requiring space-based spectroscopic data to access the most distant objects (Maiolino \& Mannucci 2018; Matsuoka et al. 2018). To circumvent this limitation in using optical emission lines, ultraviolet (UV; $1000<\lambda<2000 \AA$ ) lines can be used to estimate metallic- 
ities in a wide redshift range (e.g. Nagao, Maiolino \& Marconi 2006; Matsuoka et al. 2009; Dors et al. 2014; Pérez-Montero \& Amorín 2017; Mignoli et al. 2019). Moreover, UV narrow lines are not significantly affected by gas shocks (Matsuoka et al. 2009), which even with low velocities $\left(v \lesssim 400 \mathrm{~km} \mathrm{~s}^{-1}\right)$ are present in the narrow-line regions (NLRs) of AGNs (Dors et al. 2015; Contini 2017).

One of the first abundance estimations in AGNs using UV emission lines was carried out by Shields (1976), who derived the N/C abundances ratio for the Quasar PKS 1756+237 located at a redshift of $z=1.72$. Later, several authors (e.g. Davidson 1977; Osmer 1980; Gaskell, Shields \& Wampler 1981; Uomoto 1984; Hamann \& Ferland 1992; Ferland et al. 1996; Dietrich \& Wilhelm-Erkens 2000; Hamann et al. 2002; Nagao et al. 2006; Shin et al. 2013; Dors et al. 2014; Feltre, Charlot \& Gutkin 2016; Yang et al. 2017; Dors et al. 2018) have estimated metallicities and/or elemental abundances (usually nitrogen abundances) in Quasars or Seyferts. These estimations have been mainly based on detailed photoionization models (e.g. Ferland et al. 1996; Dors et al. 2018) or made through diagrams containing observational and model-predicted emission-line intensities (e.g. Nagao et al. 2006; Matsuoka et al. 2009, 2018), as these methodologies are difficult to apply for a large sample of data. In this sense, general calibrations between metallicity and strong emission lines or Bayesian approach (Mignoli et al. 2019) are preferable and easily applicable.

Dors et al. (2014), using photoionization model results, proposed the first calibration between the metallicity of the gas in the NLRs of AGNs and the intensity of UV emission lines through the use of the $\mathrm{C} 43=\log [(\mathrm{C}$ IV $\lambda 1549+\mathrm{C}$ III $] \lambda 1909) / \mathrm{He}$ II $\lambda 1640]$ emissionlines ratio. This calibration is strongly dependent on the ionization degree of the emitting gas, which, once the ionizing spectrum is determined, can be estimated from the C III] $\lambda 1909 /$ C IV $\lambda 1549$ emission-lines ratio (Davidson 1972). However, as was pointed out by Dors et al. (2014), this C III]/C IV ratio is somewhat dependent on the metallicity, mainly for low ionization values.

Recently, Castro et al. (2017) presented a new methodology to calibrate the optical $N 2 O 2=\log ([\mathrm{N}$ II $] \lambda 6584 /[\mathrm{O}$ II $] \lambda 3727)$ emission-line ratio with the metallicity of NLRs of AGNs, producing a semi-empirical calibration. This methodology consists of calculating the metallicity $(Z)$ and the corresponding line ratio for a sample of type-2 AGNs through diagnostic diagrams containing both observational data and photoionization model results. This method is based on the idea proposed by Pilyugin (2000, 2001), which consists in obtaining calibrations using oxygen abundances (or metallicities) derived from direct electron temperature estimations. It seems that the $T_{\mathrm{e}}$-method does not work for AGNs (Dors et al. 2015), probably due to the necessity of applying an ionization correction factor to the oxygen abundance determinations through this method (not explored up to now in this kind of objects), and the probable presence of electron temperature fluctuations and/or shocks (neither considered in our photoionization models). However, Dors et al. (2015) showed that $\mathrm{O} / \mathrm{H}$ derived from the strong emission-line calibrations is in agreement with those (independent) estimations from abundance gradient extrapolations. Thus, the metallicity and the physical conditions of the gas would seem to be better determined applying (semi-)empirical calibrations developed through photoionization models, despite these are subject to several uncertainties (e.g. Viegas 2002; Kennicutt, Bresolin \& Garnett D. 2003).

The methodology proposed by Castro et al. (2017) has the advantage of obtaining calibrations considering the closest modelled physical conditions to the real ones for each object parametrized through diagnostic diagrams and, therefore, reducing the uncertainties in metallicity estimations. In fact, for example, for $\mathrm{H}$ II regions it is well known that, in general, oxygen abundances estimated from purely theoretical calibrations are overestimated in comparison to those derived using the $T_{\mathrm{e}}$-method (e.g. Kennicutt et al. 2003; Dors \& Copetti 2005). However, Dors et al. (2011) showed that this discrepancy can be alleviated if it is required that the photoionization models simultaneously reproduce observational line ratios sensitive to the metallicity and to the ionization degree of $\mathrm{H}$ II regions (see also Dors \& Copetti 2005; Morisset et al. 2016).

Up to now, the unique metallicity calibration for AGNs based on narrow UV line ratios seems to be the theoretical one proposed by Dors et al. (2014), which is based on the C43 index. It is possible to obtain calibrations involving other strong UV line ratios, such as the $\mathrm{N} v \lambda 1240 / \mathrm{He}$ II $\lambda 1640$ ratio, suggested by Ferland et al. (1996) as a metallicity indicator.

Keeping the above in mind, we combined observational intensities of UV narrow emission lines of type-2 AGNs compiled from the literature with photoionization model results to obtain semi-empirical calibrations between the metallicity of the gas phase and the $\mathrm{N}$ v/He II emission-line intensity ratio. We also recalibrated the $\mathrm{C} 43$ lines ratio with the metallicity. This manuscript is organized as follows: in Section 2, the description of the observational data and the photoionization models is presented; in Section 3, we present the resulting calibrations, while the discussion and conclusions of the outcome are given in Sections 4 and 5 , respectively.

\section{METHODOLOGY}

With the aim of obtaining semi-empirical calibrations between the metallicity and the $\mathrm{N} \mathrm{V} \lambda 1240 / \mathrm{He}$ II $\lambda 1640$ and C43 emissionline intensity ratios, the same methodology proposed by Castro et al. (2017) was adopted, i.e. the calibrations are derived through diagnostic diagrams containing observational and model-predicted line-intensity ratios. In what follows, the observational data and the photoionization model descriptions are presented.

\subsection{Observational data}

We compiled from the literature fluxes of the N v $\lambda 1240$, C IV $\lambda 1549$, He II $\lambda 1640$, and C III] $\lambda 1909$ emission lines produced in NLRs of type-2 AGNs. The sample of objects is mainly the one considered by Matsuoka et al. (2018) with the addition of Seyfert 2 AGNs located at low redshifts $(z \lesssim 0.04)$. In this way, our sample comprises objects in the redshift range $0 \lesssim z \lesssim 4.0$, divided in Seyfert 2 (9 objects), type- 2 quasars (6 objects), high- $z$ radio galaxies (61 objects), and radio-quiet type-2 AGNs (1 object).

In Table 1, the identification, redshift, logarithm of the $\mathrm{N} \mathrm{v}$ $\lambda 1240 / \mathrm{He}$ II $\lambda 1640, \mathrm{C}$ III] $\lambda 1909 / \mathrm{C}$ IV $\lambda 1549$, and C43 $=\log [(\mathrm{C}$ IV $\lambda 1549+\mathrm{C}$ III] $\lambda 1909) / \mathrm{He}$ II $\lambda 1640]$ emission-line intensity ratios as well as the nebular parameters derived (see below) are listed. The emission lines are not reddening corrected due to the small effect of dust extinction on metallicity and ionization degree determinations obtained from the considered emission-line ratios (Nagao et al. 2006). For some objects were not possible to estimate the error in the line ratios since the uncertainties in the measurements of the emission-line fluxes are not given in the works from which the data were compiled. The N v $\lambda 1240$ flux is only available for about 30 per cent of the objects in our sample. 
Table 1. Data of AGNs from the literature and derived parameters. Object name, redshift, logarithm of the emission-line intensity ratios, metallicity (in units of solar metallicity $\mathrm{Z} / \mathrm{Z}_{\odot}$ ), and the logarithm of the number of ionizing photons [log $\left.Q(\mathrm{H})\right]$ derived from the diagnostic diagrams shown in Fig. 3 are listed for each object. Diag. 1 refers to estimations obtained from N v/He II versus C III/C IV diagram (Fig. 3: lower panel) and Diag. 2 from C43 versus C III/C IV (Fig. 3 : upper panel). In the last two columns, the logarithm of the stellar mass (in units of the solar mass) taken from Matsuoka et al. (2018), and the references to the works from which emission-line intensities were compiled are listed.

\begin{tabular}{|c|c|c|c|c|c|c|c|c|c|c|}
\hline \multirow[b]{2}{*}{ Object } & \multirow[b]{2}{*}{ Redshift } & \multirow[b]{2}{*}{$\log (\mathrm{N}$ V/He II $)$} & \multirow[b]{2}{*}{$\mathrm{C} 43$} & \multirow[b]{2}{*}{$\log (\mathrm{C}$ III]/C IV $)$} & \multicolumn{2}{|c|}{ Diag. 1} & \multicolumn{2}{|c|}{ Diag. 2} & \multirow[b]{2}{*}{$\log \left(\frac{M}{M_{*}}\right)$} & \multirow[b]{2}{*}{ Refs. } \\
\hline & & & & & $Z / Z_{\odot}$ & $\log U$ & $Z / Z_{\odot}$ & $\log U$ & & \\
\hline \multicolumn{11}{|c|}{ Seyfert 2} \\
\hline NGC 1068 & 0.004 & $0.07 \pm 0.10$ & $0.60 \pm 0.08$ & $-0.33 \pm 0.09$ & - & - & $0.81_{-0.29}^{+0.34}$ & $-1.43_{-0.10}^{+0.16}$ & - & 1 \\
\hline NGC 5506 & 0.006 & - & $0.60 \pm 0.15$ & $-0.09 \pm 0.15$ & - & - & $0.44_{-0.12}^{+0.61}$ & $-1.72_{-0.17}^{+0.17}$ & - & 1 \\
\hline NGC 7674 & 0.029 & - & $0.57 \pm 0.15$ & $-0.15 \pm 0.19$ & - & - & $0.60_{-0.07}^{+0.81}$ & $-1.63_{-0.21}^{+0.24}$ & - & 1 \\
\hline Mrk 1388 & 0.021 & - & $0.49 \pm 0.08$ & $-0.36 \pm 0.08$ & - & - & $1.16_{-0.37}^{+0.46}$ & $-1.43_{-0.09}^{+0.11}$ & - & 1 \\
\hline MCG-3-34-64 & 0.017 & $-0.30 \pm 0.08$ & $0.32 \pm 0.10$ & $-0.30 \pm 0.11$ & $1.16_{-0.33}^{+1.40}$ & $-1.50_{-0.09}^{+0.10}$ & $1.56_{-0.64}^{+1.30}$ & $-1.52_{-0.10}^{+0.14}$ & - & 1 \\
\hline NGC 7674 & 0.029 & - & $0.64 \pm 0.14$ & $-0.15 \pm 0.14$ & - & - & $0.42_{-0.11}^{+0.61}$ & $-1.66_{-0.15}^{+0.15}$ & - & 2 \\
\hline \multicolumn{11}{|c|}{ Type-2 quasar } \\
\hline CDFS-031 & 1.603 & - & $0.41 \pm 0.04$ & $-0.36 \pm 0.06$ & - & - & $1.45_{-0.32}^{+0.34}$ & $-1.44_{-0.04}^{+0.07}$ & 11.43 & 1 \\
\hline CDFS-531 & 1.544 & - & $0.32 \pm 0.04$ & $-0.18 \pm 0.05$ & - & - & $1.18_{-0.16}^{+0.33}$ & $-1.62_{-0.05}^{+0.05}$ & 11.70 & 1 \\
\hline CXO 52 & 3.288 & $-0.45 \pm 0.10$ & $0.51 \pm 0.05$ & $-0.22 \pm 0.04$ & $1.13_{-0.48}^{+0.58}$ & $-1.58_{-0.04}^{+0.04}$ & $0.82_{-0.20}^{+0.23}$ & $-1.56_{-0.04}^{+0.04}$ & - & 1 \\
\hline \multicolumn{11}{|c|}{ High- $z$ radio galaxy } \\
\hline TN J0121+1320 & 3.517 & - & $0.21 \pm 0.02$ & $0.03 \pm 0.01$ & - & - & $1.02_{-0.07}^{+0.05}$ & $-1.83_{-0.01}^{+0.01}$ & 11.02 & 4 \\
\hline TN J0205+2242 & 3.507 & - & $0.39 \pm 0.04$ & $-0.31 \pm 0.05$ & - & - & $1.26_{-0.16}^{+0.47}$ & $-1.48_{-0.05}^{+0.04}$ & 10.82 & 4 \\
\hline MRC 0316-257 & 3.130 & - & $0.30 \pm 0.02$ & $0.11 \pm 0.02$ & - & - & $0.74_{-0.05}^{+0.07}$ & $-1.92_{-0.04}^{+0.03}$ & 11.20 & 4 \\
\hline USS 0417-181 & 2.773 & - & $0.26 \pm 0.03$ & $0.19 \pm 0.04$ & - & - & $0.63_{-0.06}^{+0.19}$ & $-2.04_{-0.04}^{+0.08}$ & - & 4 \\
\hline TN J0920-0712 & 2.758 & $-0.30 \pm 0.01$ & $0.41 \pm 0.01$ & $-0.23 \pm 0.01$ & $0.88_{-0.04}^{+0.04}$ & $-1.54_{-0.01}^{+0.01}$ & $1.10_{-0.01}^{+0.01}$ & $-1.56_{-0.01}^{+0.01}$ & - & 4 \\
\hline WN J1123+3141 ${ }^{(a)}$ & 3.221 & $0.60 \pm 0.01$ & $0.61 \pm 0.01$ & $-0.93 \pm 0.06$ & $1.86_{-0.34}^{+1.17}$ & $-0.81_{-0.01}^{+0.02}$ & - & - & $<11.72$ & 4 \\
\hline $4 \mathrm{C} 24.28^{(a)}$ & 2.913 & $0.09 \pm 0.01$ & $0.32 \pm 0.01$ & $-0.18 \pm 0.02$ & - & - & $1.17_{-0.05}^{+0.05}$ & $-1.62_{-0.02}^{+0.02}$ & $<11.11$ & 4 \\
\hline MG $0311+1532$ & 1.986 & - & 0.43 & -0.20 & - & - & 0.93 & -1.57 & - & 5 \\
\hline BRL 0310-150 & 1.769 & - & 0.57 & -0.30 & - & - & 0.86 & -1.47 & - & 5 \\
\hline USS 0355-037 & 2.153 & - & 0.13 & -0.06 & - & - & 1.34 & -1.73 & - & 5 \\
\hline USS $0448+091$ & 2.037 & - & 0.44 & 0.35 & - & - & 0.25 & -2.25 & - & 5 \\
\hline USS 0529-549 & 2.575 & - & 0.56 & 0.65 & - & - & - & - & 11.46 & 5 \\
\hline $4 C+41.17$ & 3.792 & - & 0.60 & -0.16 & - & - & 0.56 & -1.63 & 11.39 & 5 \\
\hline USS $0748+134$ & 2.419 & - & 0.34 & -0.07 & - & - & 0.92 & -1.71 & - & 5 \\
\hline USS $0828+193$ & 2.572 & - & 0.31 & 0.02 & - & - & 0.85 & -1.82 & $<11.60$ & 5 \\
\hline BRL 0851-142 & 2.468 & - & 0.33 & -0.32 & - & - & 1.71 & -1.50 & - & 5 \\
\hline TN J0941-1628 & 1.644 & - & 0.76 & -0.20 & - & - & 0.27 & -1.63 & - & 5 \\
\hline USS 0943-242 & 2.923 & -0.20 & 0.36 & -0.22 & 0.57 & -1.55 & 1.17 & -1.57 & 11.22 & 5 \\
\hline MG 1019+0534 & 2.765 & -0.56 & 0.25 & -0.32 & 2.83 & -1.47 & 2.20 & -1.49 & 11.15 & 5 \\
\hline TN J1033-1339 & 2.427 & - & 0.57 & -0.51 & - & - & 1.12 & -1.24 & - & 5 \\
\hline TN J1102-1651 & 2.111 & - & 0.20 & 0.04 & - & - & 1.01 & -1.84 & - & 5 \\
\hline USS $1113-178^{(b)}$ & 2.239 & - & 0.80 & 0.21 & - & - & - & - & - & 5 \\
\hline $3 C 256.0$ & 1.824 & -0.59 & 0.24 & -0.08 & 0.81 & -1.71 & 1.16 & -1.71 & - & 5 \\
\hline USS 1138-262 & 2.156 & - & 0.20 & 0.21 & - & - & 0.76 & -2.03 & $<12.11$ & 5 \\
\hline BRL 1140-114 & 1.935 & - & 0.50 & -0.22 & - & - & 0.84 & -1.56 & - & 5 \\
\hline 4C 26.38 & 2.608 & - & 0.29 & -0.56 & - & - & 3.06 & -1.23 & - & 5 \\
\hline MG $1251+1104$ & 2.322 & - & 0.43 & 0.23 & - & - & 0.36 & -2.10 & - & 5 \\
\hline WN J1338+3532 & 2.769 & - & 0.06 & 0.22 & - & - & 0.86 & -2.08 & - & 5 \\
\hline
\end{tabular}


Table 1 - continued

\begin{tabular}{|c|c|c|c|c|c|c|c|c|c|c|}
\hline \multirow[b]{2}{*}{ Object } & \multirow[b]{2}{*}{ Redshift } & \multirow[b]{2}{*}{$\log (\mathrm{N}$ V/He II $)$} & \multirow[b]{2}{*}{$\mathrm{C} 43$} & \multirow[b]{2}{*}{$\log (\mathrm{C}$ III]/C IV $)$} & \multicolumn{2}{|c|}{ Diag. 1} & \multicolumn{2}{|c|}{ Diag. 2} & \multirow[b]{2}{*}{$\log \left(\frac{M}{M_{*}}\right)$} & \multirow[b]{2}{*}{ Refs. } \\
\hline & & & & & $Z / Z_{\odot}$ & $\log U$ & $\mathrm{Z} / \mathrm{Z}_{\odot}$ & $\log U$ & & \\
\hline \multicolumn{11}{|c|}{ High- $z$ radio galaxy } \\
\hline MG 1401+0921 & 2.093 & - & 0.17 & -0.08 & - & - & 1.27 & -1.72 & & 5 \\
\hline 3C 294 & 1.786 & -0.69 & 0.34 & 0.07 & - & - & 0.72 & -1.89 & 11.36 & 5 \\
\hline USS $1410-001$ & 2.363 & -0.17 & 0.24 & -0.47 & 1.78 & -1.34 & 3.05 & -1.32 & $<11.41$ & 5 \\
\hline USS $1425-148$ & 2.349 & - & 0.15 & -0.36 & - & - & 3.37 & -1.42 & - & 5 \\
\hline USS $1436+157$ & 2.538 & - & 0.64 & -0.25 & - & - & 0.60 & -1.51 & - & 5 \\
\hline 3C 324.0 & 1.208 & - & 0.42 & -0.02 & - & - & 0.70 & -1.77 & - & 5 \\
\hline USS $1558-003$ & 2.527 & - & 0.36 & -0.35 & - & - & 1.65 & -1.47 & $<11.70$ & 5 \\
\hline BRL 1602-174 & 2.043 & - & 0.42 & -0.56 & - & - & 1.85 & -1.25 & - & 5 \\
\hline TXS J1650+0955 & 2.510 & - & 0.21 & -0.42 & - & - & 3.15 & -1.37 & - & 5 \\
\hline 8C $1803+661$ & 1.610 & - & 0.44 & -0.44 & - & - & 1.46 & -1.35 & - & 5 \\
\hline $4 C 40.36$ & 2.265 & - & 0.33 & -0.02 & - & - & 0.87 & -1.77 & 11.29 & 5 \\
\hline BRL 1859-235 & 1.430 & - & 0.24 & 0.14 & - & - & 0.81 & -1.95 & - & 5 \\
\hline $4 C 48.48$ & 2.343 & - & 0.38 & -0.33 & - & - & 1.52 & -1.49 & - & 5 \\
\hline MRC 2025-218 ${ }^{(a)}$ & 2.630 & 0.24 & 0.67 & 0.14 & - & - & - & - & $<11.62$ & 5 \\
\hline TXS J2036+0256 & 2.130 & - & 0.41 & 0.30 & - & - & 0.35 & -2.17 & - & 5 \\
\hline MRC 2104-242 & 2.491 & - & 0.53 & -0.15 & - & - & 0.66 & -1.62 & 11.19 & 5 \\
\hline $4 C 23.56$ & 2.483 & -0.04 & 0.34 & -0.21 & - & - & 1.17 & -1.59 & 11.59 & 5 \\
\hline MG $2121+1839$ & 1.860 & - & 0.74 & -0.34 & - & - & 0.52 & -1.40 & - & 5 \\
\hline USS 2251-089 & 1.986 & - & 0.56 & -0.34 & - & - & 0.92 & -1.43 & - & 5 \\
\hline MG 2308+0336 ${ }^{(a)}$ & 2.457 & 0.16 & 0.44 & -0.14 & - & - & 0.85 & -1.64 & - & 5 \\
\hline $4 C 28.58^{(b)}$ & 2.891 & - & 0.11 & 0.77 & - & - & 0.24 & -2.71 & 11.36 & 5 \\
\hline MP J0340-6507 & 2.289 & - & $0.18 \pm 0.11$ & $0.22 \pm 0.16$ & - & - & $0.77_{-0.36}^{+0.47}$ & $-2.05_{-019}^{+0.19}$ & - & 6 \\
\hline TN J1941-1951 & 2.667 & - & $0.43 \pm 0.12$ & $-0.65 \pm 0.22$ & - & - & $2.08_{-1.03}^{+0.05}$ & $-1.18_{-0.20}^{+0.29}$ & - & 6 \\
\hline $\begin{array}{l}\text { MPerrorJdot2352- } \\
6154\end{array}$ & 1.573 & - & $0.39 \pm 0.06$ & $-0.36 \pm 0.08$ & - & - & $1.51_{-0.43}^{+0.56}$ & $-1.46_{-0.06}^{+0.11}$ & - & 6 \\
\hline
\end{tabular}

COSMOS 05162

3.524

$0.78 \pm 0.05 \quad-0.46 \pm 0.07$

$0.46_{-0.17}^{+0.21} \quad-1.25_{-0.13}^{+0.16}$

10.50

7

Notes. ${ }^{(a)}$ Observational data located out of the region occupied by the model results in the $\mathrm{N}$ v/He II versus $\mathrm{C}$ III/C IV diagram (see Fig. 3 , lower panel). ${ }^{(b)}$ Observational data located out of the region occupied by the model results in the C43 versus C III/C IV diagram (see Fig. 3, upper panel). References: (1) Nagao et al. (2006), (2) Kraemer et al. (1994), (3) Díaz, Prieto \& Wamsteker (1988), (4) Matsuoka et al. (2009), (5) De Breuck et al. (2000), (6) Bornancini et al. (2007), and (7) Matsuoka et al. (2018).

\subsection{Photoionization models}

The photoionization models were built using the CLOUDY code version 17.00 (Ferland et al. 2017) in order to compare the predicted UV emission-line intensity ratios with those measured for the type2 AGNs in our sample. The range of the input values for the photoionization model parameters is similar to the ones used by Dors et al. (2018) and in what follows we described them.

The predicted emission-line intensities of our models for the NLRs of type-2 AGNs are mainly driven by four parameters: the ionization parameter $(U)$, the metallicity $(Z)$, the electron density $\left(N_{\mathrm{e}}\right)$, and the shape of the spectral energy distribution (SED). $U$ is defined as $U=Q_{\text {ion }} / 4 \pi R_{\text {in }}^{2} N c$, where $Q_{\text {ion }}$ is the number of hydrogen ionizing photons emitted per second by the ionizing source, $R_{\text {in }}$ is the distance from the ionization source to the inner surface of the ionized gas cloud (in $\mathrm{cm}$ ), $N$ is the particle density (in $\mathrm{cm}^{-3}$ ), and $c$ is the speed of light. Excitation differences owing to variations in the mass of the gas phase, or in the geometrical conditions covering a wide range of possible scenarios, are covered by photoionization models assuming variations of the ionization parameter (Pérez-Montero 2014). We consider $U$ in the range $-3.5 \leqq \log U \leqq-0.5$, with a step of $0.5 \mathrm{dex}$; almost the same values considered by Feltre et al. (2016) and Castro et al. (2017).

Regarding the electron density, Dors et al. (2014) found that the NLRs of Seyfert 2 galaxies present gas with low electron density values, $N_{\mathrm{e}} \lesssim 3000 \mathrm{~cm}^{-3}$ (see also Revalski et al. 2018a,b). We assumed in our models a constant electron density along the radius and equal to $N_{\mathrm{e}}=500 \mathrm{~cm}^{-3}$, an averaged value derived by Dors et al. (2014). The outer radius $\left(R_{\text {out }}\right)$ was chosen as the one for which the electron temperature reaches $4000 \mathrm{~K}$, since cooler gas practically does not produce UV emission lines. The resulting geometry is plane-parallel. It is worth mentioning that models with different combinations of $Q_{\mathrm{ion}}, R_{\mathrm{in}}$, and $N_{\mathrm{e}}$ but that result in the same $U$ are homologous models with about the same predicted emission-line intensities (Bresolin, Kennicutt \& Garnett 1999).

The SED was considered to be composed by two components: one representing the Big Blue Bump peaking at 1 Ryd, and the other a power law with a spectral index $\alpha_{\mathrm{x}}=-1$ representing the nonthermal X-ray radiation. As usual, the continuum between $2 \mathrm{keV}$ and $2500 \AA$ is described by a power law with the spectral index

$\alpha_{\mathrm{ox}}=\frac{\log [F(2 \mathrm{kev}) / F(2500 \AA)]}{\log [v(2 \mathrm{keV}) / v(2500 \AA)]}$,

where $F$ is the flux at the given frequency $v$ (Tananbaum et al. 1979). Miller et al. (2011) combined observational data of about 700 radiointermediate and radio-loud quasars in a wide range of wavelengths and derived $\alpha_{\text {ox }}$ in the range $-2.0 \lesssim \alpha_{\text {ox }} \lesssim-0.8$ (see also Zamorani et al. 1981). A histogram containing the distribution of the values found by Miller et al. (2011) is presented in Fig. 1. We can see that the high $\left(\alpha_{\mathrm{ox}}>-1\right)$ and low $\left(\alpha_{\mathrm{ox}}<-1.8\right)$ values are derived for few ( $\sim 3$ per cent) objects. The averaged $\alpha_{\text {ox }}$ is $-1.37 \pm 0.16$. We consider in the models $\alpha_{\text {ox }}=-1.4$, about the same averaged value calculated from estimations by Miller et al. (2011).

Regarding the metallicity, Dors et al. (2014) found that the C43 index is bivaluated, yielding one lower branch for $\left(Z / Z_{\odot}\right) \lesssim 0.2$ and 


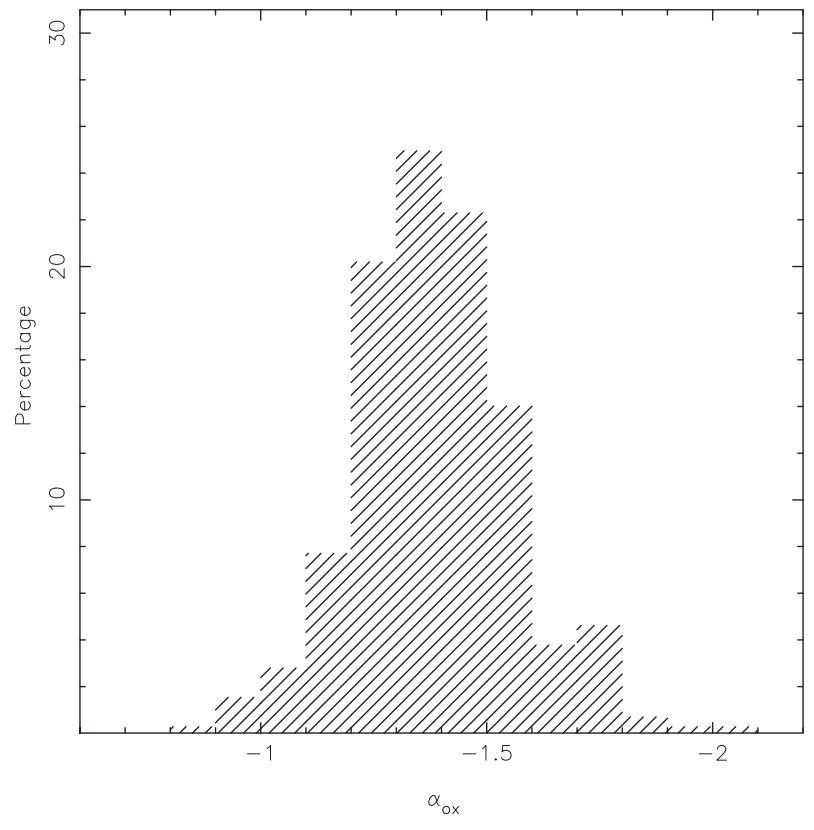

Figure 1. Histogram containing the distribution of the spectral index $\alpha_{\mathrm{ox}}$ derived by Miller et al. (2011) for about 700 radio-intermediate and radioloud quasars.

an upper branch for $\left(Z / Z_{\odot}\right) \gtrsim 0.2$. This problem is also present in the classical $Z-R_{23}$ calibration (Pagel et al. 1979) for star-forming regions and this degeneracy can be broken by using the [N II] $\lambda 6584 /[\mathrm{O}$ II] $\lambda 3727$ emission-line ratio (Kewley \& Ellison 2008). Due to the small number of measurements of UV emission lines in most of the AGN spectra, the degeneracy in C43 and N v/He II calibrations can only be eliminated based on physical reasons. Studies of the NLRs of nearby Seyfert 2 AGNs (e.g. StorchiBergmann et al. 1998; Groves, Heckman \& Kauffmann 2006; Dors et al. 2015; Castro et al. 2017; Revalski et al. 2018a,b; Thomas et al. 2018) and of objects at high redshift (e.g. Nagao et al. 2006; Dors et al. 2014; Matsuoka et al. 2009, 2018) have showed that metallicities of AGNs are generally higher than $\left(Z / Z_{\odot}\right)=0.2$. Cosmological simulations (see fig. 10 of Dors et al. 2014) and chemical evolution models (Mollá \& Díaz 2008) do not predict low metallicities for the nuclear regions of galaxies (including AGNs). Besides, Matsuoka et al. (2018) derived a relation between the stellar mass of galaxies containing type-2 AGNs and their NLR metallicities, which were derived using a comparison between the observational intensities of $\mathrm{C}$ IV/He II and C III]/He II line ratios and their photoionization model-predicted values. These authors found two metallicity values for galactic stellar masses $\log \left(M_{\star} / \mathrm{M}_{\odot}\right)$ between 11 and 11.5 , and they pointed out that the lowest metallicity solution $\left(Z / Z_{\odot} \lesssim 0.2\right)$ is implausible since a significant decrease of metallicity with the stellar mass would be obtained. As these authors remarked, this behaviour has not been reported for any galaxy population at any redshift, and has not been reproduced by any model or cosmological simulation. Therefore, we adopted only metallicity values higher than $\left(Z / Z_{\odot}\right)=0.2$ for our AGN models, i.e. we estimated the metallicities using the upper branch of the relation of the metallicity with the C43 index and with $\mathrm{N}$ v/He II emission-line ratio. Assuming a solar oxygen abundance $12+\log (\mathrm{O} / \mathrm{H})_{\odot}=8.69$ (Alende Prieto, Lambert \& Asplund 2001), we considered the following values for the metallicity in relation to the solar one $\left(Z / Z_{\odot}\right): 0.2,0.5,0.75,1.0,1.5,2.0$, and 4.0.
All elements except nitrogen, carbon, and helium are taken to be primary nucleosynthesis elements. For the helium abundance, we used the relation obtained from chemical abundance determinations of H II regions by Dopita et al. (2006):

$\mathrm{He} / \mathrm{H}=0.0737+0.024 \times\left(Z / \mathrm{Z}_{\odot}\right)$.

Regarding the nitrogen and carbon secondary nucleosynthesis elements, their abundance in relation to the oxygen abundance (or metallicity) is poorly known for AGNs. In fact, the unique quantitative nitrogen abundance estimation for AGNs is the one performed by Dors et al. (2017) for a small sample of Seyfert 2 galaxies. The predicted $\mathrm{C} 43$ and $\mathrm{N}$ v/He II ratios are dependent on the assumed $\mathrm{C}-\mathrm{O}$ and $\mathrm{N}-\mathrm{O}$ relations, respectively. Hence, the derived metallicities are also dependent on the assumed relations. Thus, we performed several simulations in order to try to estimate these relations.

First, we built a grid of models assuming a fixed value of $\alpha_{\text {ox }}=-1.4$ and $N_{\mathrm{e}}=500 \mathrm{~cm}^{-3}$, while $Z$ and $\log U$ range in the values described above. In this grid, the $\mathrm{N}-\mathrm{O}$ relation obtained by Dors et al. (2017)

$$
\log (\mathrm{N} / \mathrm{H})=1.05( \pm 0.09) \times[\log (\mathrm{O} / \mathrm{H})]-0.35( \pm 0.33)
$$

for nearby Seyfert 2 AGNs, and the $\mathrm{C}-\mathrm{O}$ relation

$(\mathrm{C} / \mathrm{H})=6.0 \times 10^{-5} \times\left(\mathrm{Z} / \mathrm{Z}_{\odot}\right)+2.0 \times 10^{-4} \times\left(\mathrm{Z} / \mathrm{Z}_{\odot}\right)^{2}$

obtained by Dopita et al. (2006) for H II regions were assumed. This grid is referred as Grid-1.

In Fig. 2 (left-hand panels), the diagrams $\mathrm{N}$ v/He II versus C III]/C IV (lower panel) and C43 versus C III]/C IV (upper panel) containing the observational data listed in Table 1 and the photoionization model results (Grid-1) are shown. We can see that the predicted intensities of $\mathrm{N}$ V/He II and $\mathrm{C} 43$ are not in agreement with the observed ones.

Secondly, we adopt a linear scale between the carbon and oxygen abundances

$(\mathrm{C} / \mathrm{H})=\left(\mathrm{Z} / \mathrm{Z}_{\odot}\right) \times(\mathrm{C} / \mathrm{H})_{\odot}$.

For the nitrogen, the relation

$(\mathrm{N} / \mathrm{H})=\left(Z / \mathrm{Z}_{\odot}\right)^{2} \times(\mathrm{N} / \mathrm{H})_{\odot}$,

given by Hamann \& Ferland (1993), was considered. This relation for the broad-line gas belonging to quasi-stellar objects was derived applying spectral synthesis and chemical enrichment models (see also Hamann et al. 2002). We assumed $\log (\mathrm{N} / \mathrm{H})_{\odot}=-4.07$ and $\log (\mathrm{C} / \mathrm{H})_{\odot}=-3.61$ taken from Holweger (2001) and Alende Prieto, Lambert \& Asplund (2002), respectively. The results of this second set of photoionization models (referred as Grid-2) are also shown in Fig. 2 (right-hand panels), where it can be seen that with the exception of few points, the carbon line ratios (upper panel) are well reproduced by the models but they fail to reproduce the observational $\mathrm{N}$ V/He II (lower panel). Therefore, we performed a new grid of models assuming

$\mathrm{N} / \mathrm{H}=A \times\left(\mathrm{Z} / \mathrm{Z}_{\odot}\right)^{n} \times(\mathrm{N} / \mathrm{H})_{\odot}$

and $A$ ranging from 1 to 5.0 and $n$ ranging from 1 to 2 . The results of these new grids were compared with the observational line ratios in two diagrams (not shown): $\mathrm{N}$ V/He II and $\mathrm{C} 43$ versus $\mathrm{C} \mathrm{III]/C} \mathrm{IV.} \mathrm{We}$ found that $A=4.5$ and $n=1.2$ produce model results compatible with the observational data, in the sense that the model results fit most of the observational data. The results of this grid are shown in Fig. 3. It must be noted that the $\mathrm{N}$ V/He II predictions are almost 

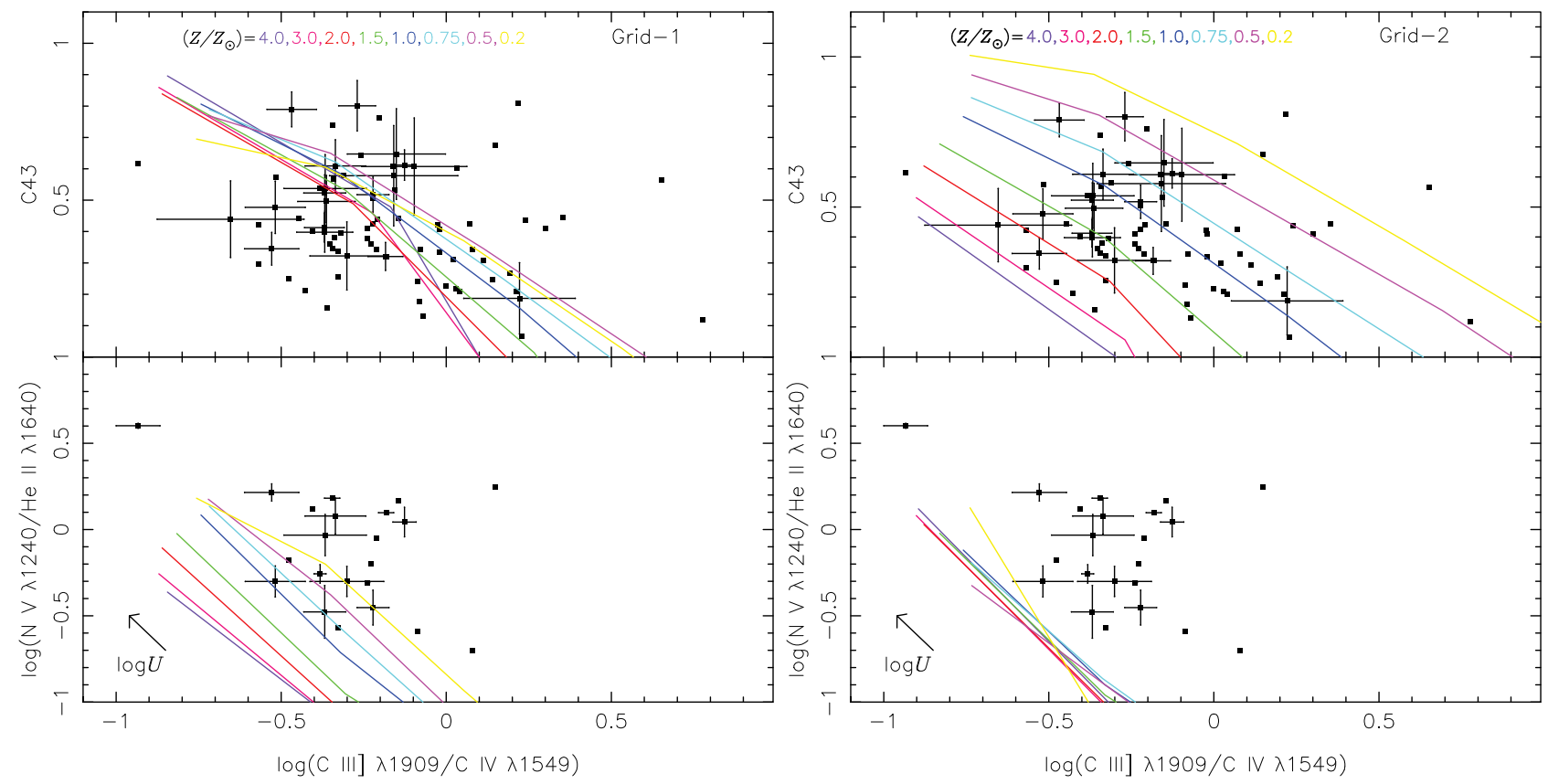

Figure 2. Diagrams containing observational data and photoionization model results. The points represent the observational data listed in Table 1 . The curves represent the photoionization model results described in Section 2.2, where the solid lines connect models with equal metallicity $(Z)$ as indicated. Left-hand panel: photoionization model results assuming the $\mathrm{N}-\mathrm{O}$ (equation 3) and C-O (equation 4) relations proposed by Dors et al. (2017) and Dopita et al. (2006), respectively. The arrow indicates the direction in which the number of ionizing photons increases in the models. Right-hand panel: same as the left-hand panel but assuming the $\mathrm{N}-\mathrm{Z}$ relation (equation 6) proposed by Hamann \& Ferland $(1993)$ and $\mathrm{C} / \mathrm{H}=(\mathrm{Z} / \mathrm{Z})_{\odot} \times(\mathrm{C} / \mathrm{H})_{\odot}$.

independent of the carbon abundance assumed in the models, such as $\mathrm{C} 43$ is independent of the nitrogen abundances.

In Fig. 4, top panel, we compare the logarithm of N/O abundance ratio as a function of $12+\log (\mathrm{O} / \mathrm{H})$ (which traces the metallicity) assumed in our models $\left(\mathrm{N} / \mathrm{H}=4.5 \times\left(\mathrm{Z} / \mathrm{Z}_{\odot}\right)^{1.2} \times(\mathrm{N} / \mathrm{H})_{\odot}\right)$ with estimations for NLRs of Seyfert 2 galaxies at $z<0.1$ from Dors et al. (2017), for $\mathrm{H}$ II regions calculated through the $T_{\mathrm{e}}$-method by Esteban et al. (2002, 2009, 2014), García-Rojas \& Esteban (2007), López-Sánchez et al. (2007), Berg et al. (2016), and Pilyugin \& Grebel (2016), ${ }^{1}$ and for the broad-line regions (BLRs) of quasars derived by Uomoto (1984) through detailed photoionization models. Since Uomoto (1984) did not derive the metallicity, i.e. only the $\mathrm{N} / \mathrm{C}$ and $\mathrm{O} / \mathrm{C}$ abundance ratios were estimated, his estimations are presented by a hatched area. We can see that our assumed values are in agreement with those for BLRs of quasars and they are higher than the ones for Seyfert 2 and $\mathrm{H}$ II regions.

Also in Fig. 4, bottom panel, we compare the $\log (\mathrm{C} / \mathrm{O})$ as a function of $12+\log (\mathrm{O} / \mathrm{H})$ assumed in our models, (i.e. a fixed value $\log (\mathrm{C} / \mathrm{O})=-0.50$ ) with values for $\mathrm{H}$ II regions and BLRs of quasars taken from the same references as in the upper panel. Unfortunately, there are no carbon abundance estimation for NLRs of Seyfert 2 galaxies in the literature. We can see that the $\mathrm{C}-\mathrm{O}$ relation assumed in our models is in consonance with the ones for BLRs and $\mathrm{H}$ II regions. A C/O abundance fixed to the solar value was also considered by Feltre et al. (2016), who built a wide grid of

${ }^{1}$ Pilyugin \& Grebel (2016) used the C-method (Pilyugin, Grebel \& Mattsson 2012), which produces similar values than the ones obtained by the $T_{\mathrm{e}}$ method.

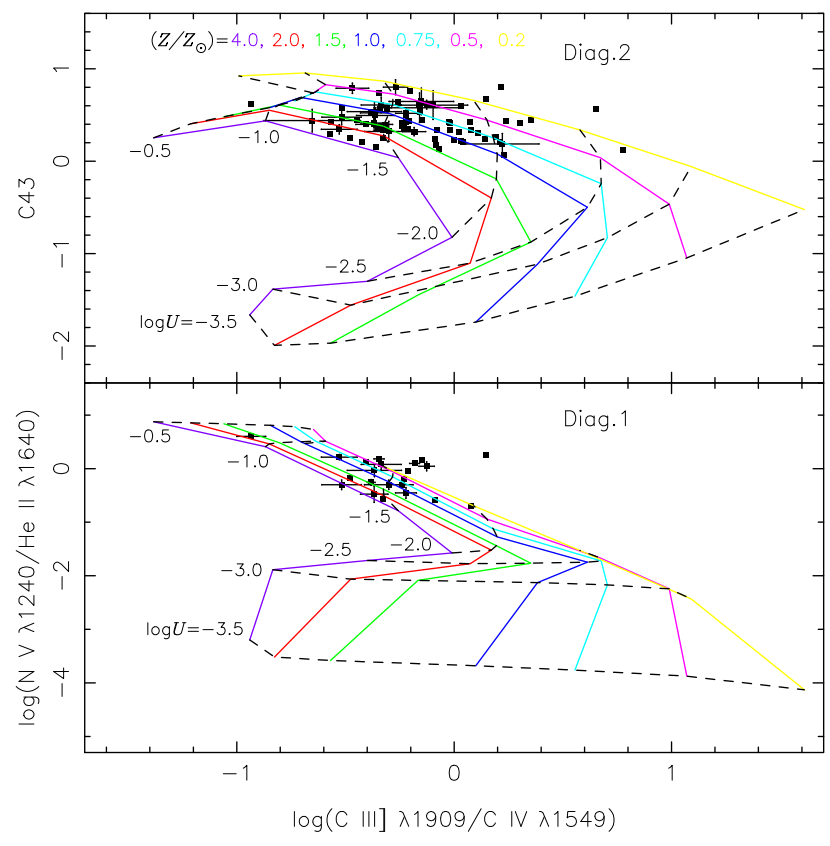

Figure 3. Diagrams containing observational data and the best solution for the photoionization model results, i.e. assuming in the models the relations $\mathrm{N} / \mathrm{H}=4.5 \times\left(\mathrm{Z} / \mathrm{Z}_{\odot}\right)^{1.2} \times(\mathrm{N} / \mathrm{H})_{\odot}$ and $\mathrm{C} / \mathrm{H}=\left(\mathrm{Z} / \mathrm{Z}_{\odot}\right) \times(\mathrm{C} / \mathrm{H})_{\odot}$ (see Section 2.2). The points represent the observational data listed in Table 1. The curves represent the photoionization model results described in Section 2.2, where the solid lines connect models with equal metallicity $(Z)$ and the dashed lines connect models with equal number of ionizing photons, as indicated. 


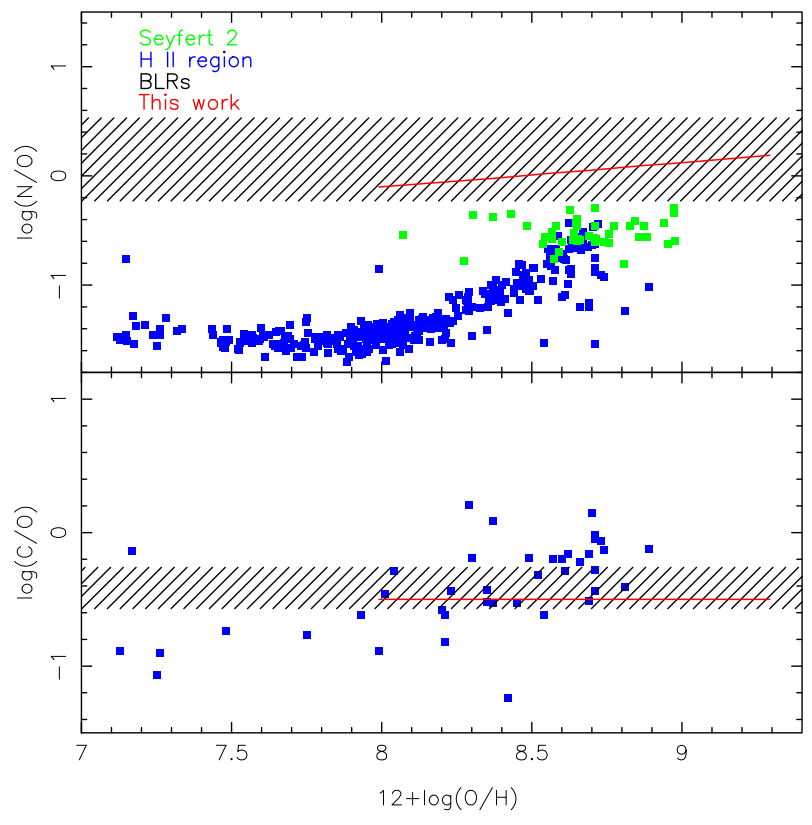

Figure 4. $\log (\mathrm{N} / \mathrm{O})$ and $\log (\mathrm{C} / \mathrm{O})$ versus $12+\log (\mathrm{O} / \mathrm{H})$ for different kinds of objects, as indicated. The green points represent abundance ratios for NLRs of Seyfert 2 AGNs at $z<0.1$ from Dors et al. (2017). The blue points represent direct estimations of abundance ratios for star-forming regions taken from Esteban et al. (2002, 2009, 2014), García-Rojas \& Esteban (2007), López-Sánchez et al. (2007), Berg et al. (2016), and Pilyugin \& Grebel (2016). The hatched areas represent the range of $\log (\mathrm{N} / \mathrm{O})$ and $\log (\mathrm{C} / \mathrm{O})$ abundance values derived for BLRs of quasars and based on detailed photoionization models by Uomoto (1984). The red lines represent the relations assumed in our models.

models in order to identify new line-ratio diagnostics to discriminate between gas photoionized by AGNs and by hot main-sequence stars as well as to estimate the metallicity (see also Nagao et al. 2006; Dors et al. 2014; Matsuoka et al. 2018).

As was mentioned above, we consider a fixed value for the electron density $\left(N_{\mathrm{e}}=500 \mathrm{~cm}^{3}\right)$. Nevertheless, the electron density varies in this kind of objects (Zhang et al. 2013; Dors et al. 2014), producing uncertainties in $Z$ estimations based on strong emission lines. In fact, Feltre et al. (2016) showed that the location of their models in diagrams containing predicted strong optical and UV emission-line ratios varies when different $N_{\mathrm{e}}$ values are assumed. Also, Nagao et al. (2006) found that metallicities based on UV emission lines are larger when high electron density values $\left(\sim 10^{5} \mathrm{~cm}^{-3}\right)$ are assumed in photoionization models instead of low electron density values $\left(\lesssim 10^{3} \mathrm{~cm}^{-3}\right)$. In order to test the $N_{\mathrm{e}}$ influence on our $Z$ estimations, first, we construct grids of models assuming $N_{\mathrm{e}}=100,500$, and $3000 \mathrm{~cm}^{-3}$ (the range of values found for NLRs of type-2 AGNs by Dors et al. 2014) constant along the AGN radius. The results of these grids were compared with the observational data (see Section 2.1) in two diagrams (not shown) $\mathrm{N}$ v/He II and C43 versus $\mathrm{C}$ III]/C IV and the interpolated $Z / \mathrm{Z}_{\odot}$ value for each object of the sample was obtained. In Fig. 5, a comparison of these metallicity estimations is shown. It can be seen that slightly higher metallicity values are derived when higher $N_{\mathrm{e}}$ values are assumed in the models. However, the discrepancies between the estimations are of the order of the uncertainties of these. Secondly, we consider models with the density ranging along the AGN radius. Revalski et al. (2018a) obtained spatially resolved optical data of the nuclear region of the Seyfert 2 galaxy Markarian 573. These authors found a density profile in the NLR of this object, with a central electron density peak of about $3000 \mathrm{~cm}^{-3}$ and a decrease in this value following a shallow power law, i.e. $N_{\mathrm{e}} \propto r^{-0.5}, r$ being the distance to the AGN centre. We built photoionization models assuming this radial density profile (not shown) and the intensities of UV emission-lines predicted by these are about the same ones of models with $N_{\mathrm{e}}$ constant along $r$. Therefore, electron density variations have an almost negligible influence in the considered line ratios for our estimations.

Finally, we also explore the dependence of the spectral index $\alpha_{\mathrm{ox}}$ on our metallicity estimations. To do this, we built grids of photoionization models assuming three different index values: $\alpha_{\mathrm{ox}}=-2.0,-1.4$, and -0.8 . This is the range of $\alpha_{\mathrm{ox}}$ values found by the observational study carried out by Miller et al. (2011) and represented in Fig. 1. The SED with $\alpha_{\text {ox }}=-2.0$ represents an ionizing source with a very soft spectrum, yielding models with very low ionization degree and, hence, with emission-line ratios largely discrepant from those of the observational sample. It must be noted that very few ( $\lesssim 1$ per cent) objects studied by Miller et al. (2011) have $\alpha_{\text {ox }}$ near to -2.0 . The results of these grids were compared with the observational data in two diagrams (not shown) $\mathrm{N}$ V/He II and $\mathrm{C} 43$ versus $\mathrm{C}$ III]/C IV and the interpolated $Z / Z_{\odot}$ values were estimated for each object. In Fig. 6, a comparison of these metallicity estimations is shown. It can be seen that when $\alpha_{\mathrm{ox}}=-0.8$ is considered higher $(\sim 90$ per cent $)$ metallicity values are obtained in comparison with those estimated by models with $\alpha_{\mathrm{ox}}=-1.4$. However, as can be seen in Fig. 1, very hard SEDs $\left(\alpha_{\text {ox }}<-1.0\right)$ are derived only for few $(\sim 2$ per cent) objects and most part of AGNs presents $\alpha_{\text {ox }}$ around -1.4. Therefore, we conclude that our metallicity estimations based on the models with $\alpha_{\mathrm{ox}}=-1.4$ (see Fig. 3) seem to relate to the real metallicity of the observed regions.

Thomas et al. (2018) presented a Bayesian code that implements a general method of comparing observed emission-line fluxes to photoionization model grids representing AGNs (see also Blanc et al. 2015) and taking into account a large range of nebular parameters (e.g. gas pressure, depletions on to dust grains) and SEDs of the ionizing source. Basically, this method determines the probability of that a set of model parameter values is representative of a set of observational data of a given object. Applying this method, Thomas, Kewley \& Dopita (2019) found a correlation between the mass of the host galaxy and the metallicity of the AGN (see also Matsuoka et al. 2018) for a sample from the SDSS (York et al. 2000). Thomas et al. (2019) pointed out that their sophisticated analysis produces almost an identical mass-metallicity relation to the one derived from the Z-N2O2 calibration obtained by Castro et al. (2017) and based on similar photoionization models (and methodology) applied in the present work (see also Mignoli et al. 2019). Therefore, metallicity estimations based on simplified photoionization models seem to be reliable.

\section{RESULTS}

The diagrams in Fig. 3, $\mathrm{N}$ v/He II versus $\mathrm{C} \mathrm{III]/C} \mathrm{IV} \mathrm{(Diag.} \mathrm{1,}$ lower panel) and C43 versus C III]/C IV (Diag. 2, upper panel) were used to calculate the logarithm of the number of ionizing photons $\left[\log (Q(\mathrm{H})]\right.$ and the metallicity $\left(Z / \mathrm{Z}_{\odot}\right)$ for each object in our sample applying a linear interpolation between the models. These results are listed in Table 1. The interpolated parameters together with the observational line ratios for each object were used to obtain semi-empirical calibrations.

In Fig. 7 (upper panel), the bi-parametric calibration $\left(\mathrm{Z} / \mathrm{Z}_{\odot}\right)=f(\mathrm{~N} \mathrm{v} / \mathrm{He} \mathrm{II}, \mathrm{C} \mathrm{III]} / \mathrm{C} \mathrm{IV})$ is shown. Its expression is as 

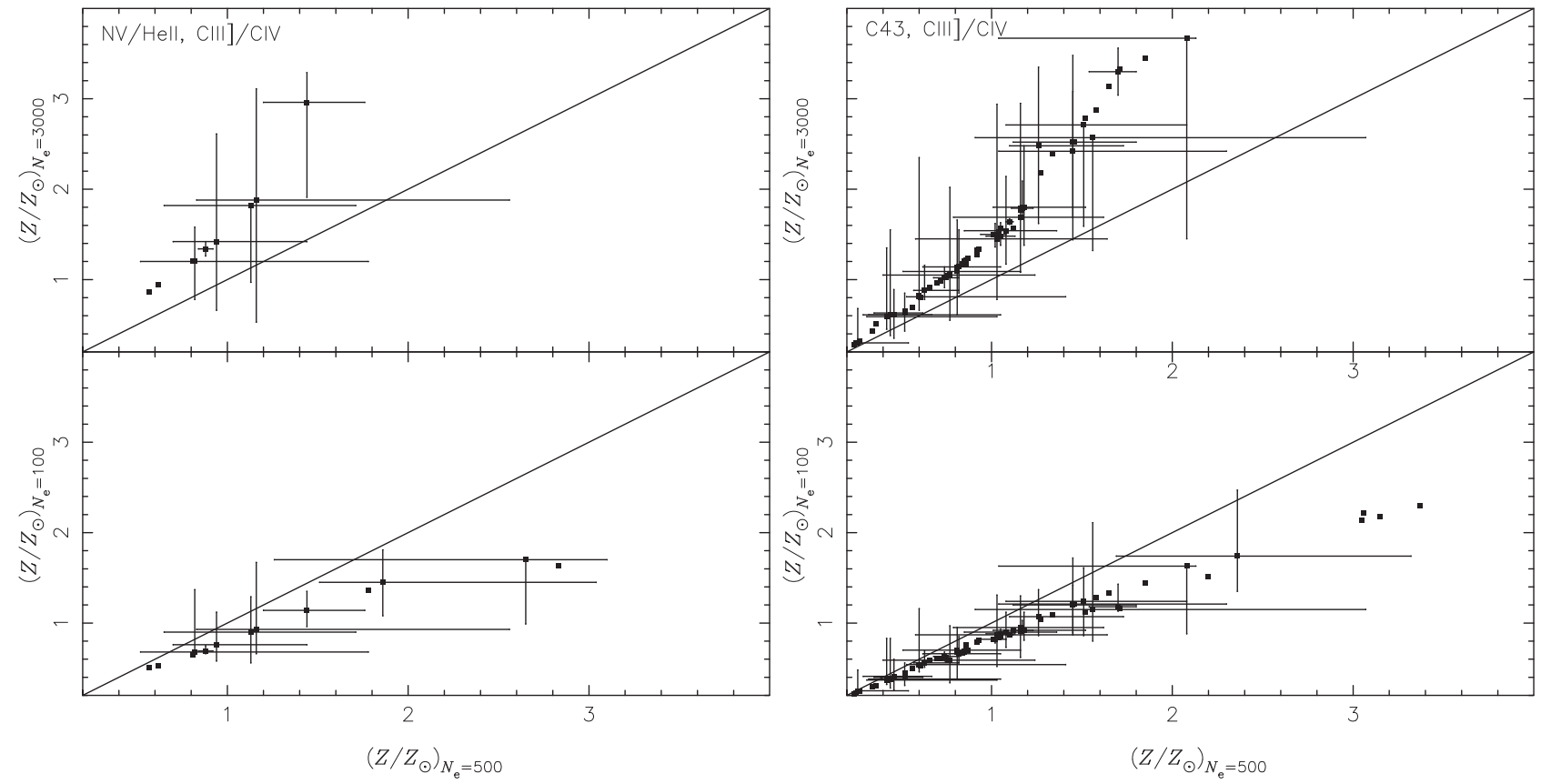

Figure 5. Comparison between metallicity $\left(Z / Z_{\odot}\right)$ estimations for the sample of objects (see Section 2.1) obtained from the diagrams (not shown) $\mathrm{N}$ v/He II versus C III]/C IV (left-hand panel) and C43 versus C III]/C IV (right-hand panel) considering grid of models assuming electron density $N_{\mathrm{e}}=100,500$, and $3000 \mathrm{~cm}^{-3}$, as indicated in the axis labels.
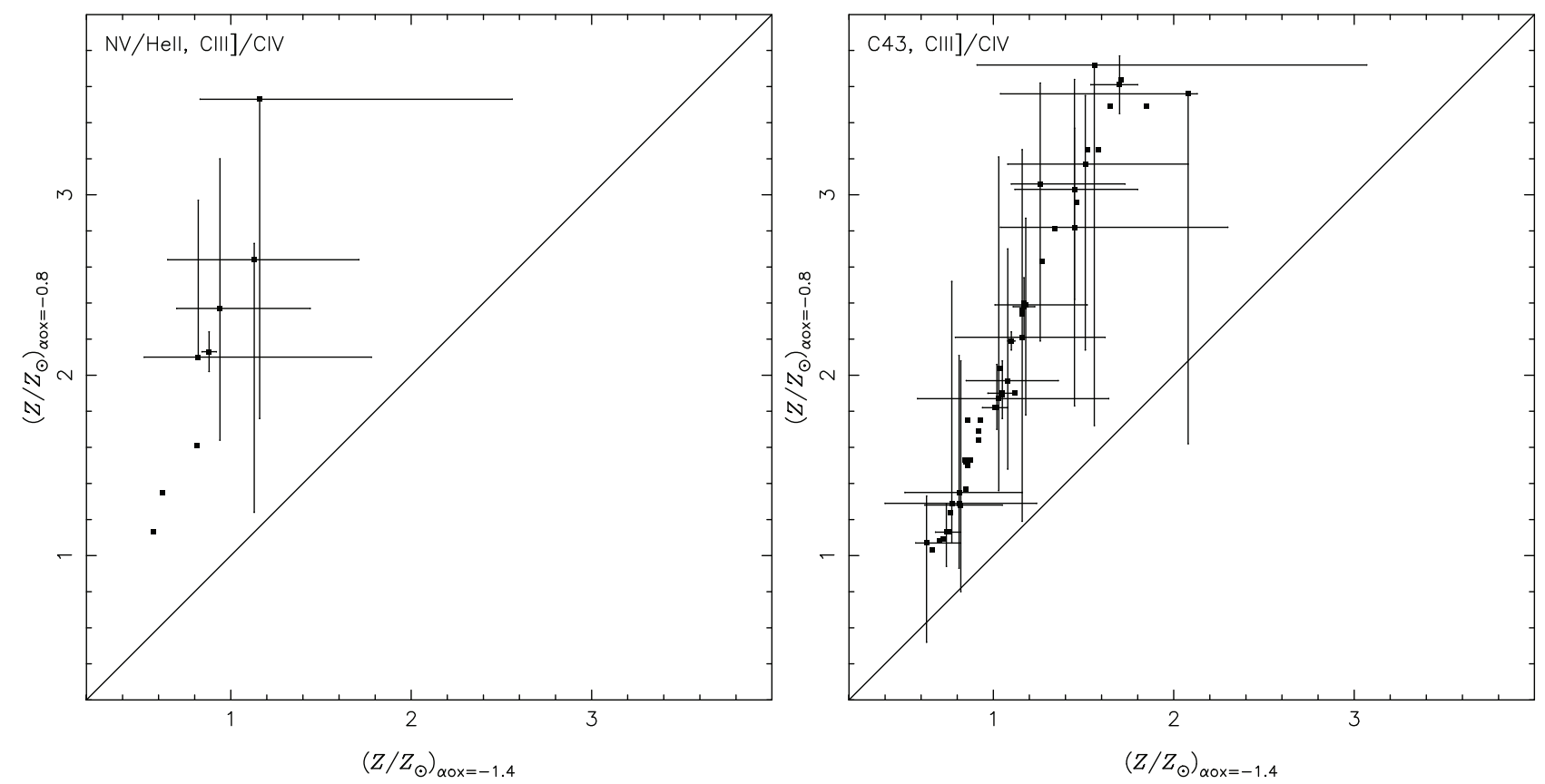

Figure 6. Same as Fig. 5 but for grids of models assuming $\alpha_{\mathrm{ox}}$ equal to -1.4 and -0.8 .

follows:

$$
\begin{aligned}
& \left(Z / Z_{\odot}\right)=(1.48 \pm 0.69)+(12.61 \pm 3.23) x^{2}+(6.28 \pm 0.78) y^{2} \\
& +(17.66 \pm 3.08) x y+(6.75 \pm 3.03) x \\
& +\left(5.50_{ \pm 1.44) y,}\right.
\end{aligned}
$$

where $\quad x=\log (\mathrm{C}$ III $] / \mathrm{C}$ IV $)$ and $y=\log (\mathrm{N} \mathrm{V} / \mathrm{He}$ II $)$. In the same way, the result for the bi-parametric calibration
$\left(\mathrm{Z} / \mathrm{Z}_{\odot}\right)=f(\mathrm{C} 43, \mathrm{CIII}] / \mathrm{C}$ IV) is shown in Fig. 7 (right-hand panel). The explicit form for this second calibration is as follows:

$$
\begin{aligned}
& \left(Z / Z_{\odot}\right)=(2.13 \pm 0.09)+(2.41 \pm 0.19) x^{2}+(4.76 \pm 0.58) C 43^{2} \\
& +\left(7.79_{ \pm 0.59) x} C 43-(4.64 \pm 0.19) x\right. \\
& -(5.64 \pm 0.48) C 43
\end{aligned}
$$




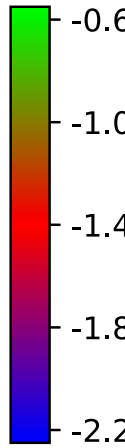

$-0.6$

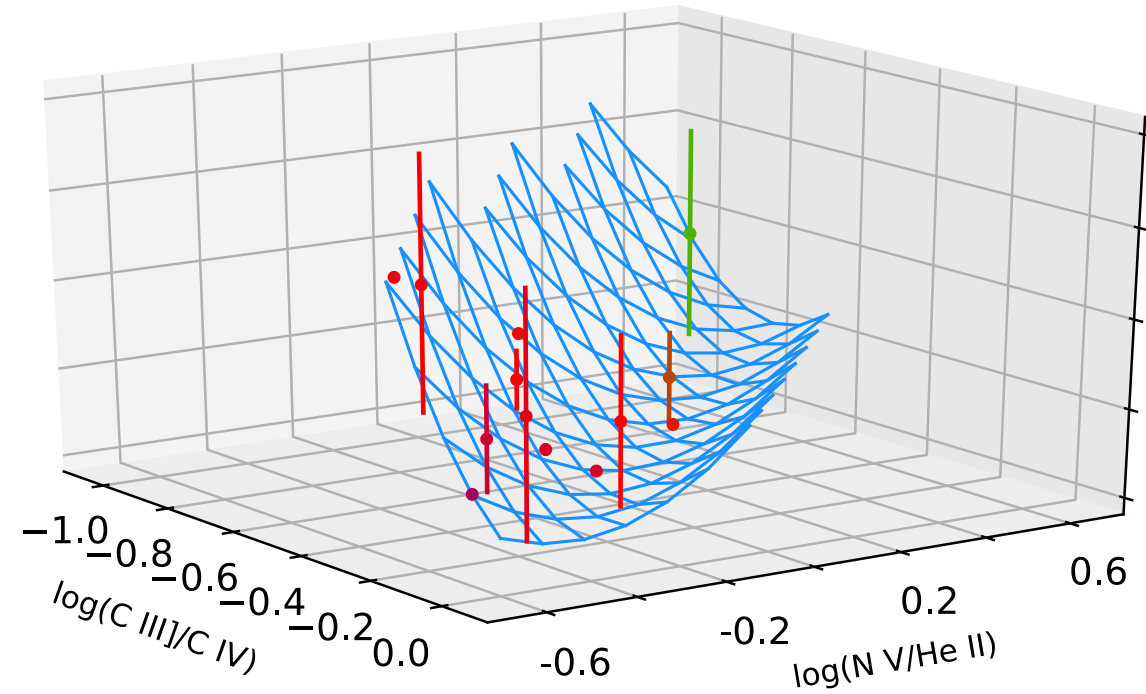

4.0

3.0

$\log U$

0.0

$2.0 \stackrel{\circ}{\mathrm{N}}$

1.0

In Fig. 8, a calibration between the logarithm of the ionization parameter and the $\mathrm{C}$ III]/C IV emission-line ratio is shown. The linear regression obtained is as follows:

$\log U=-(0.14 \pm 0.02) \times x^{2}-\left(1.10_{ \pm 0.01}\right) \times x-\left(1.80_{ \pm 0.01}\right)$.

In Fig. 9, the logarithms of the ionization parameter obtained with the diagnostic diagrams presented in Fig. 3 (Diag. 1 and Diag. 2) and listed in Table 1 are compared. This figure shows a very good agreement between both estimations.

\section{DISCUSSION}

\subsection{Metallicity in narrow- and broad-line regions}

The first metallicity estimations in BLRs of AGNs based on UV emission lines were obtained by Shields (1976) and Davidson
(1977), who used the initial photoionization models (see Davidson 1972; Shields 1974; Baldwin \& Netzer 1978) to investigate which combinations of line ratios are the most appropriate to estimate abundance ratios (e.g. N/C and O/C). Later, Uomoto (1984) obtained UV spectra of a sample of six quasars $(1.6<z<2.1)$ and, fitting photoionization models to the observational line measurements, Uomoto found about solar oxygen abundances and a slight enhancement of nitrogen abundances for two of these quasars. Other studies, as for example Hamann et al. (1997), concluded that BLRs, in fact, present supersolar abundances $\left(Z>5 Z_{\odot}\right)$ and an enhancement of $\mathrm{N}$ abundances (see also Peimbert 1968; Alloin 1973; Storchi-Bergmann \& Pastoriza 1990; Storchi-Bergmann 1991; Dietrich et al. 2003; Bradley, Kaiser \& Baan 2004). More recently, Sameshima, Yoshii \& Kawara (2017) derived the metallicity, traced by the $\mathrm{Fe} / \mathrm{H}$ and $\mathrm{Mg} / \mathrm{Fe}$ abundance ratios, in the BLRs of a sample of about 17500 quasars $(0.7 \lesssim z \lesssim 1.7)$, whose 


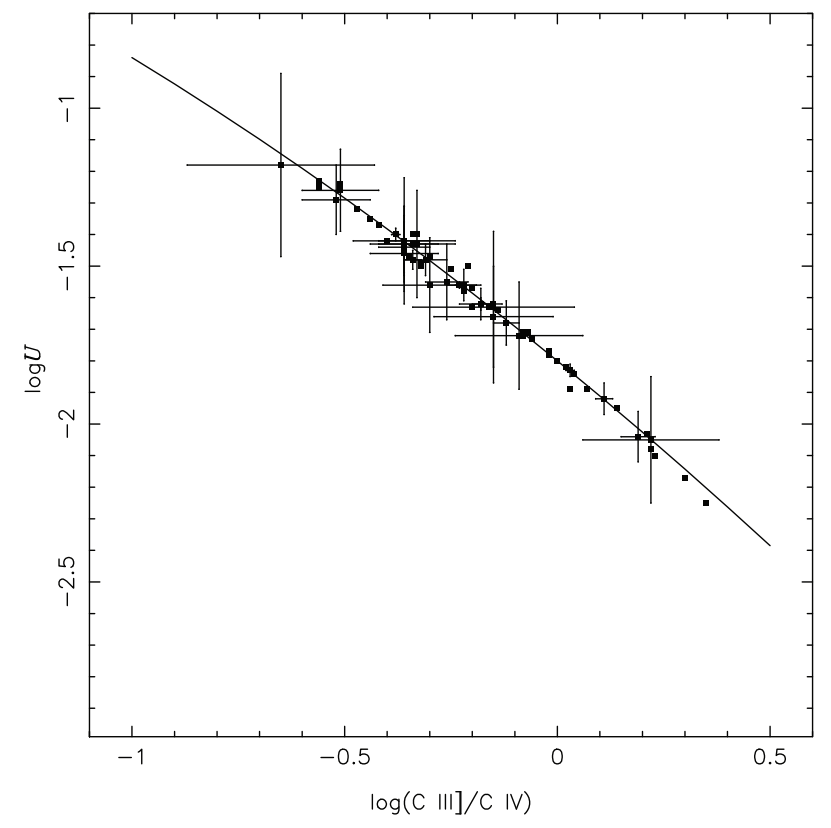

Figure 8. As in Fig. 7 but for $\log U$ as a function of the logarithm of C III] $\lambda 1909 / \mathrm{C}$ IV $\lambda 1549$ emission-line ratio. The line represents the best fit.

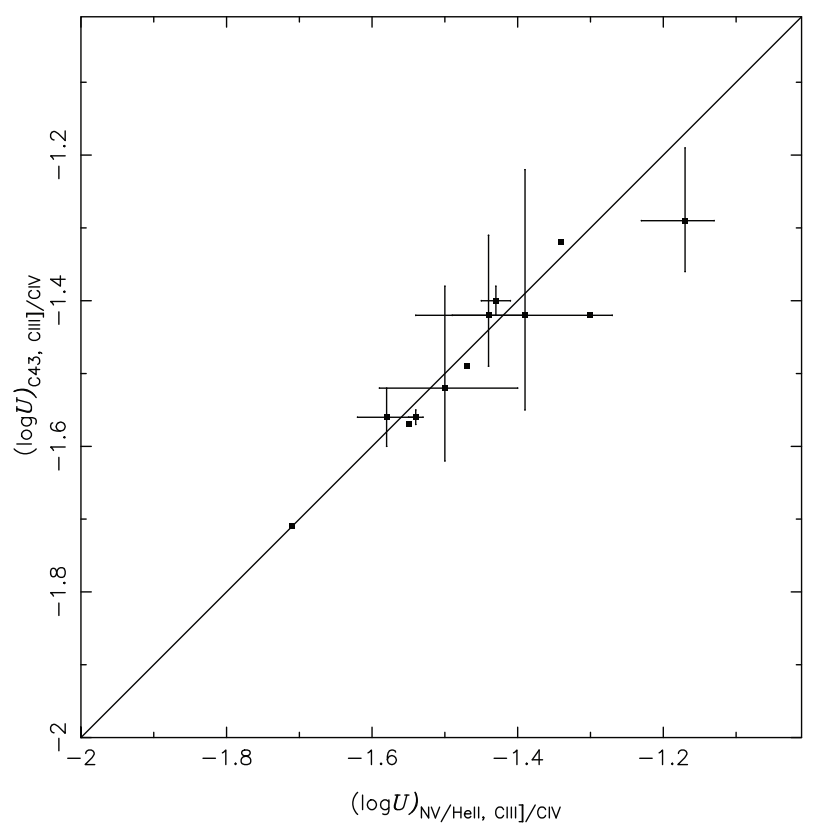

Figure 9. Comparison between $\log U$ obtained with Diag. 2 versus $\log U$ obtained with Diag. 1 (see Fig. 3) and listed in Table 1. The solid line represents the one-to-one relation.

observational data were taken from the SDSS (York et al. 2000). To obtain the metallicity, these authors compared observational fluxes and equivalent widths of Fe II (calculated by integrating the fitted Fe II template in the 2200-3090 $\AA$ wavelength range) and $\mathrm{Mg}$ II $\lambda 2798$ lines with photoionization model results. They found that the majority of the objects have oversolar metallicities with a median value of $Z \approx 3 Z_{\odot}$, reaching up to $\left(Z / Z_{\odot}\right) \sim 100$ (see also Dietrich \& Wilhelm-Erkens 2000; Hamann et al. 2002; Batra \& Baldwin 2014).

In order to compare our NLRs $Z$ estimations with those for BLRs, a histogram containing the metallicity distribution for our

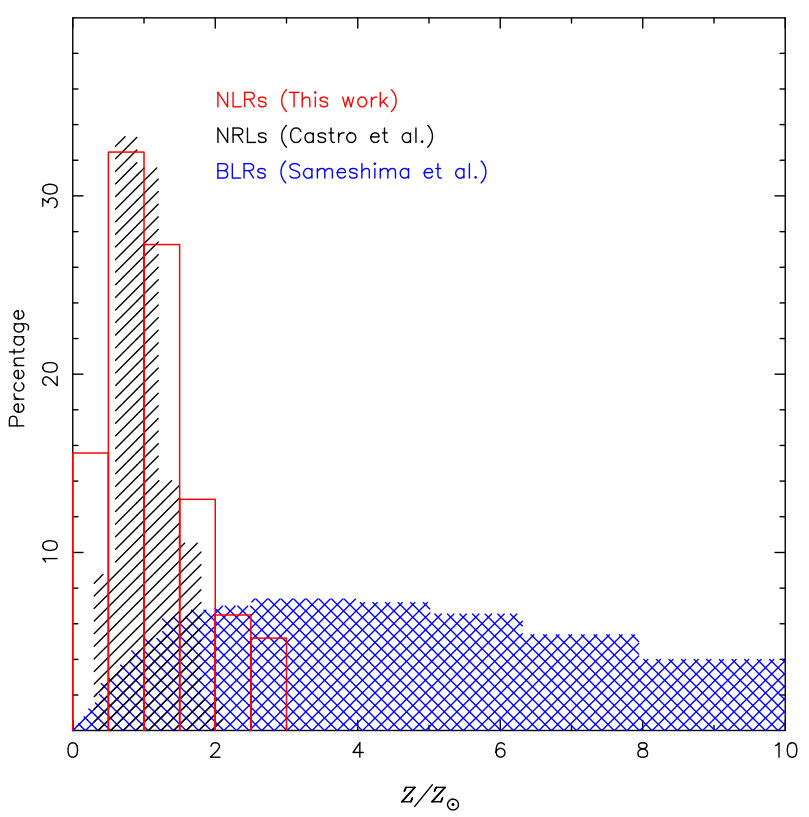

Figure 10. Histograms containing NLRs and BLRs metallicity distributions. The red (empty-filled) histogram represents NLRs metallicity distribution for our sample $(0.04<z<4.0)$ and using the equation (8). The black histogram (with slant-filled pattern) represents NLRs metallicity distribution for a sample of Seyfert 2 AGNs $(z<0.1)$ derived using a semiempirical calibration proposed by Castro et al. (2017). The Cyan histogram (with $\mathrm{x}$-filled pattern) represents BLRs metallicity distribution for a sample of quasars $(1.6<z<2.1)$ derived by Sameshima et al. (2017).

sample, derived using equation (8), and the results of Sameshima et al. (2017) is presented in Fig. 10. We limited their estimations to $\left(Z / Z_{\odot}\right) \leqq 10$ since few objects present higher metallicities. In Fig. 10, the NLR metallicity results derived by Castro et al. (2017), from a semi-empirical calibration between $Z$ and the optical ratio $N 2 O 2=\log ([\mathrm{N} \mathrm{II}] \lambda 6584 /[\mathrm{O}$ II $] \lambda 3727)$, are also shown. We found that the majority ( $\sim 60$ per cent) of the NLRs metallicity estimations based on the $\mathrm{C} 43$ are in the $0.5 \lesssim\left(Z / \mathrm{Z}_{\odot}\right) \lesssim 1.5$ range, with a median value of $<Z / Z_{\odot}>\sim 1.1$. Very different metallicity distributions are found for NLRs and BLRs, the latter being more extended and with a median value higher, by a factor of $2-3$, than the former. This difference could be probably due to the fact that broad lines originate in a small region with a radius lower than 1 pc (Kaspi et al. 2000; Suganuma et al. 2006), which may evolve more rapidly than the NLRs (Matsuoka et al. 2018). As can be seen in Fig. 10, our metallicity estimations follow the same distribution than those derived by Castro et al. (2017) for the NLRs of Seyfert 2 AGNs, who found metallicities in the $0.7 \lesssim\left(Z / Z_{\odot}\right) \lesssim 1.2$ range for most of their objects.

\subsection{Metallicity estimations from distinct calibrations}

Previous studies of metallicities in AGNs (e.g. Shemmer \& Netzer 2002; Dietrich et al. 2003; Dhanda et al. 2007) and in star-forming regions (e.g. Kewley \& Ellison 2008; López-Sánchez \& Esteban 2009; Dors et al. 2011) have shown that different methods or different calibrations of the same index provide different metallicity values, with discrepancies of up to 1.0 dex.

In order to compare the estimations from our two calibrations, in the bottom panel of Fig. 11, $Z$ estimations for the 


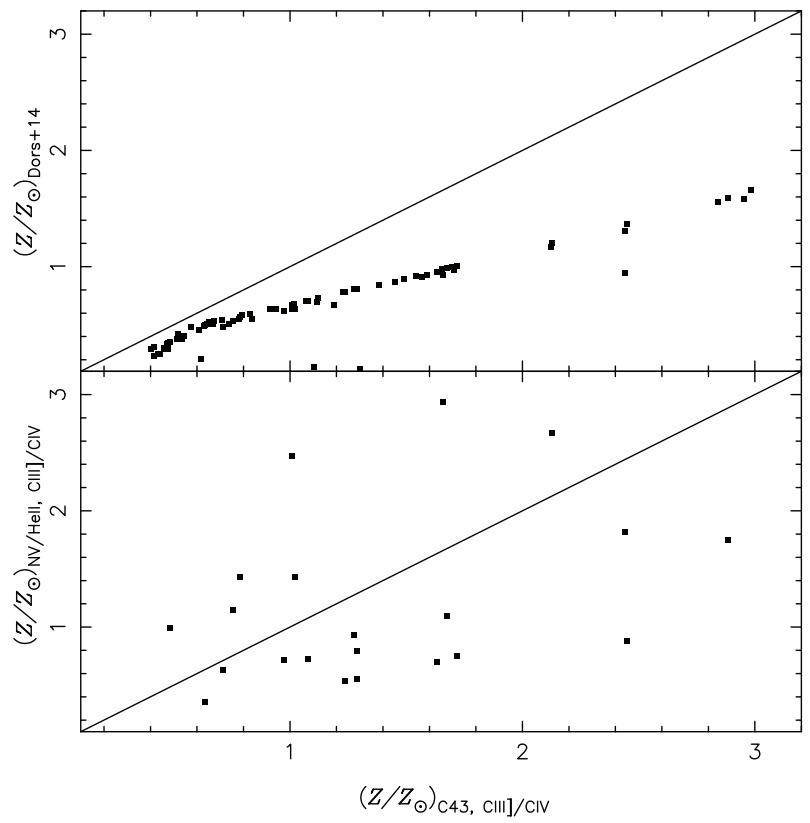

Figure 11. Comparison between NLRs metallicity estimations for the objects in our sample obtained through distinct calibrations. The solid lines represent the one-to-one relation between the estimations. Bottom panel: estimations via $Z / \mathrm{Z}_{\odot}=f(\mathrm{Nv} / \mathrm{He}$ II, $\mathrm{C}$ III] $/ \mathrm{C}$ IV) (equation 7) versus those via $Z / Z_{\odot}=f(\mathrm{C} 43, \mathrm{CIII}] / \mathrm{C}$ IV) (equation 8 ). Upper panel: estimations via the theoretical calibration $\left(Z / \mathrm{Z}_{\odot}\right)=f(\mathrm{C} 43, \mathrm{C} \mathrm{III]} / \mathrm{C} \mathrm{IV})$ proposed by Dors et al. (2014) versus those via $\left.Z / Z_{\odot}=f(C 43, \mathrm{C} \mathrm{III}] / \mathrm{C} \mathrm{IV}\right)$ (equation 8).

objects in our sample obtained from the bi-parametric calibration $\left(\mathrm{Z} / \mathrm{Z}_{\odot}\right)=f(\mathrm{~N} \mathrm{v} / \mathrm{He}$ II, $\mathrm{C} \mathrm{III]} / \mathrm{C} \mathrm{IV})$ (equation 7$)$ are plotted against the estimations via $\left(Z / Z_{\odot}\right)=f(C 43, \mathrm{CIII}] / \mathrm{C}$ IV) (equation 8 ). It can be seen that, despite the large scattering and the few number of points, the estimations are in consonance with each other.

In the top panel of Fig. 11, the estimations from the semiempirical calibration $\left(\mathrm{Z} / \mathrm{Z}_{\odot}\right)=f(\mathrm{C} 43, \mathrm{C} \mathrm{III}] / \mathrm{C}$ IV) (equation 8) are compared to those derived via the theoretical relation by Dors et al. (2014), where it can be seen that the later one produces $Z$ values higher than the former, mainly for the high-metallicity regime. This difference is probably due to Dors et al. (2014) did not deeply explore the dependence between the metallicity and the $U-\mathrm{C}$ III/C IV relation. Since the semi-empirical and bi-parametric calibrations (equations 7 and 8) are derived taken into account observational data, they are, in principle, more robust and confident than the purely theoretical calibration derived by Dors et al. (2014).

Finally, we compared our UV estimations for individual objects with the ones based on optical emission lines. Unfortunately, only for three objects in our sample it was possible this comparison: the Seyfert 2 galaxies: NGC 5506, Mrk 3, and Mrk 573. We used the optical emission-line intensities of these galaxies, compiled by Dors et al. (2015), and estimated the metallicity through the theoretical calibration between $\mathrm{Z}$ and $N 2 O 2$ index proposed by Castro et al. (2017):

$$
\begin{aligned}
\left(\mathrm{Z} / \mathrm{Z}_{\odot}\right)= & 1.08( \pm 0.19) \times N 2 O 2^{2} \\
& +1.78( \pm 0.07) \times N 2 O 2+1.24( \pm 0.01)
\end{aligned}
$$

and through the first calibration proposed by Storchi-Bergmann et al. (1998, hereafter SB98):

$$
\begin{aligned}
(\mathrm{O} / \mathrm{H})_{\mathrm{SB} 98}= & 8.34+(0.212 x)-\left(0.012 x^{2}\right)-(0.002 y) \\
& +(0.007 x y)-\left(0.002 x^{2} y\right)+\left(6.52 \times 10^{-4} y^{2}\right) \\
& +\left(2.27 \times 10^{-4} x y^{2}\right)+\left(8.87 \times 10^{-5} x^{2} y^{2}\right)
\end{aligned}
$$

where $x=[\mathrm{N} \mathrm{II}] \lambda \lambda 6548,6584 / \mathrm{H} \alpha, y=[\mathrm{O}$ III $] \lambda \lambda 4959,5007 / \mathrm{H} \beta$, and

$$
\left(\mathrm{Z} / \mathrm{Z}_{\odot}\right)_{\mathrm{SB} 98}=10^{\left[(\mathrm{O} / \mathrm{H})_{\mathrm{SB} 98}-8.69\right]} .
$$

The estimations using these optical calibrations are compared with the ones via equations (7) and (8) in Table 2. We can see that the $f(\mathrm{C} 43, \mathrm{C} \mathrm{III}] / \mathrm{C}$ IV) relation (equation 8 ) produces the closest values to the ones from the optical calibrations (equations 10 and 11). Nevertheless, it must be noted that only three objects do not constitute a statistically significant comparison sample.

\subsection{Mass-Metallicity relation}

Recently, for the first time, Matsuoka et al. (2018) showed that there is a dependence between the NLR metallicities of type-2 AGNs $(1.2<z<4.0)$ with the stellar masses of their host galaxies, in the sense that AGNs with higher metallicities are located in more massive galaxies. These authors divided the sample in bins of stellar masses $\left(M_{*} / \mathrm{M}_{\odot}\right)$ and, for each bin, calculated NLR metallicities by comparing the $\mathrm{C}$ IV/He II and $\mathrm{C}$ III/C IV ratios of the averaged observed emission-line fluxes with those predicted by photoionization models. Matsuoka et al. (2018) did not derive an expression for the stellar mass-metallicity $(M-Z)$ relation. In order to verify if our $Z$ estimations indicate the existence of an $M-Z$ relation and to derive an expression for it, the $Z$ estimations obtained from equations (7) and (8) are plotted in Fig. 12 (lower and upper panel, respectively) as a function of the stellar mass for those galaxies in our sample with this parameter available in the literature (listed in Table 1). It must be noted that all these galaxies are in $1.6<z<3.8$. Although there are few objects with determinations of the stellar masses of the host galaxy, we can see that the metallicity values estimated from equation (7) increase with the galaxy mass, obtaining

$$
\begin{aligned}
\left(Z / \mathrm{Z}_{\odot}\right)_{\mathrm{NV} / \mathrm{He} \mathrm{II}, \mathrm{C} \mathrm{III} / \mathrm{C} \mathrm{IV}}= & {\left[1.39 \pm 1.36 \times \log \left(M_{*} / \mathrm{M}_{\odot}\right)\right] } \\
& -(14.33 \pm 15.43) .
\end{aligned}
$$

The $M-Z$ relation via equation (8) is represented by

$$
\left(Z / \mathrm{Z}_{\odot}\right)_{\mathrm{C} 43, \mathrm{C} \text { III] } / \mathrm{C} \mathrm{IV}}=\left[0.32 \pm 0.38 \times \log \left(M_{*} / \mathrm{M}_{\odot}\right)\right]
$$

$$
-(2.49 \pm 4.31) \text {. }
$$

The Pearson correlation coefficients $(P)$ for the $M-Z$ relations based on equations (7) and (8) are 0.32 and 0.18 , respectively, indicating a positive but weak correlation. It can be seen in the upper panel of Fig. 12 that there is only one object with $\log \left(M_{*} / \mathrm{M}_{\odot}\right)>12$ and this mass value is an upper limit. Removing this point from the fitting, we obtained

$$
\begin{aligned}
\left(Z / \mathrm{Z}_{\odot}\right)_{\mathrm{C} 43, \mathrm{C} \text { III } / \mathrm{C} \mathrm{IV}}= & {\left[0.56 \pm 0.42 \times \log \left(M_{*} / \mathrm{M}_{\odot}\right)\right] } \\
& -(5.24 \pm 4.76)
\end{aligned}
$$

and $P=0.28$.

Unfortunately, as can be seen in the lower panel of the Fig. 12, we have a small number of objects with metallicities derived from 
Table 2. Object names and their corresponding metallicities $\left(Z / Z_{\odot}\right)$ obtained through the indicated equations.

\begin{tabular}{lcccc}
\hline Object & $\begin{array}{c}\mathrm{Z} / \mathrm{Z}_{\odot} \\
(\mathrm{N} \mathrm{V/He} \mathrm{II,C} \mathrm{III]/C} \mathrm{IV)} \\
\text { (Equation 7) }\end{array}$ & $\begin{array}{c}f(\mathrm{C} 43, \mathrm{C} \text { III } / \mathrm{C} \text { IV) } \\
\text { (Equation 8) }\end{array}$ & $\begin{array}{c}\text { N2O2 } \\
\text { (Equation 10) }\end{array}$ & $\begin{array}{c}\text { SB98 } \\
\text { (Equation 12) }\end{array}$ \\
\hline NGC 5506 & - & 0.47 & 1.15 & 0.81 \\
Mrk 3 & 2.47 & 1.00 & 1.16 & 1.14 \\
Mrk573 & 1.09 & 1.67 & 1.12 & 0.91 \\
\hline
\end{tabular}

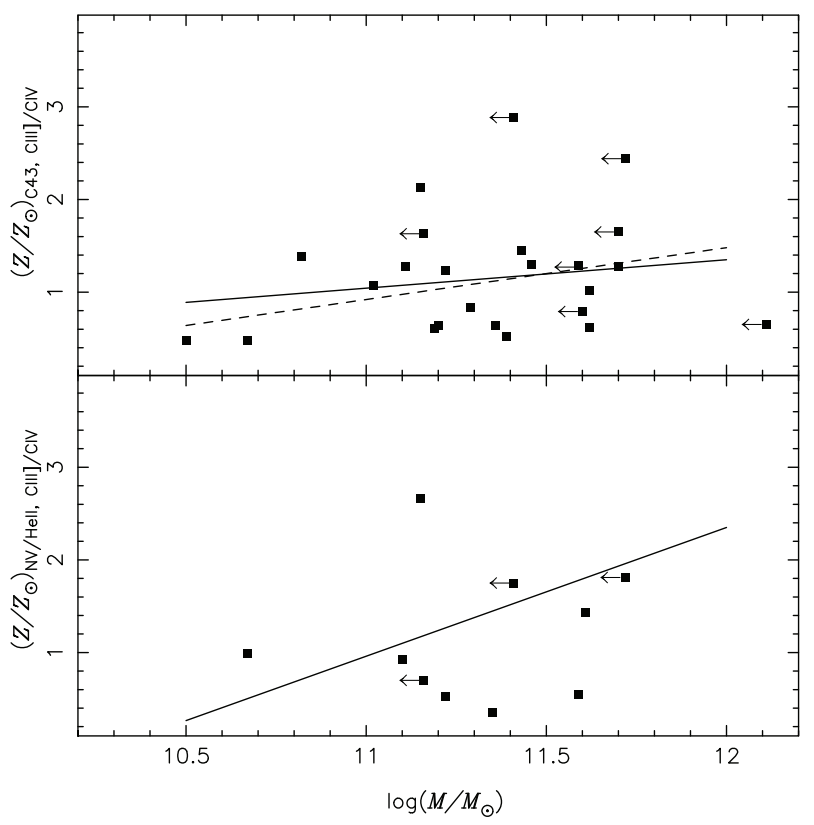

Figure 12. $M-Z$ relation for type-2 AGNs. Metallicity values were calculated via equation (7) (bottom panel) and equation (8) (upper panel). The solid lines represent the linear regressions (equations 13 and 14). In the upper panel, the dashed line represents the linear regression without taking into account the more massive object (equation 15). The arrows indicate that only the upper limit of the stellar mass was quoted in the literature (see Table 1).

equation (7) and with estimations of their stellar masses. Hence, we consider that the $M-Z$ relation given by equation (13) is not statistically significant; hereafter, we will only discuss the $M-Z$ relation given by equation (15).

In Fig. 13, the $M-Z$ relation given by equation (15) is compared with the relation obtained by Thomas et al. (2019) for local $(z<0.2)$ Seyfert 2 AGNs (in the mass interval $10.1<\left[\log \left(M_{*} / \mathrm{M}_{\odot}\right)\right]<11.3$ ), and with $M-Z$ relations for star-forming galaxies at different redshift ranges derived by Maiolino et al. (2008). We can see a difference between our $M-Z$ relation and the one derived by Thomas et al. (2019). Taking into account the very different redshift ranges between the Thomas and collaborators and our samples, this difference in the $M-Z$ relation could be explained by the evolution of this relation with the redshift. This interpretation is based on the observed gradual declination of the metallicity with the redshift for a given mass in SF galaxies from the Maiolino and collaborators work as can be seen in Fig. 13 (see also Savaglio et al. 2005; Erb et al. 2006; Yabe et al. 2014; Ly et al. 2016). It is noticeable that our relation (equation 15) seems to complement the one derived by Maiolino et al. (2008) for $z \sim 3.5$ towards higher masses. It is worth emphasizing that a larger sample of AGNs with metallicity

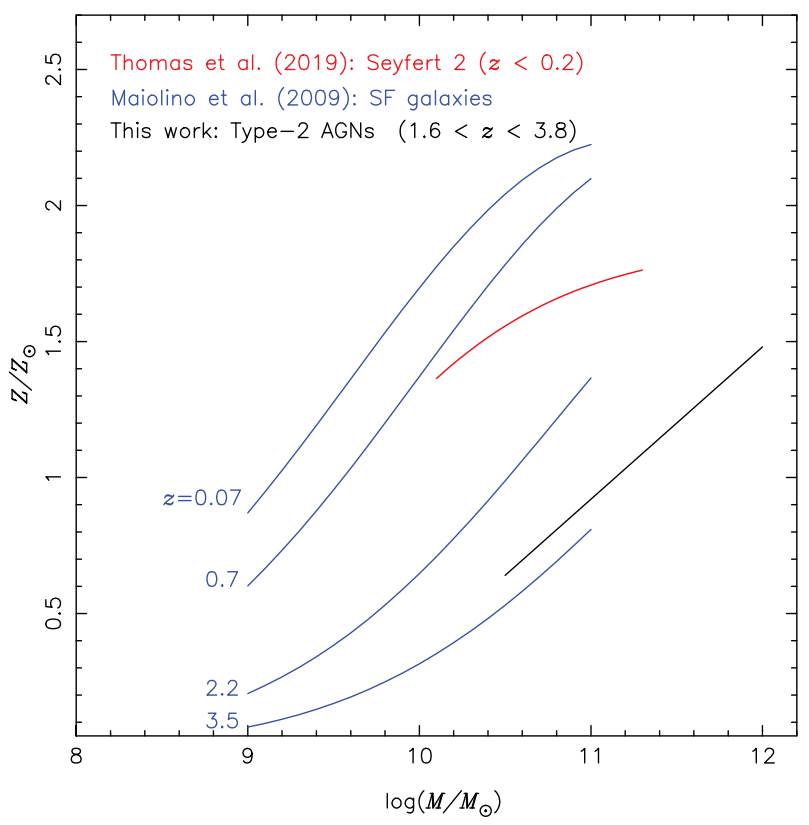

Figure 13. Comparison between our $M-Z$ relation given by equation (15) and the relations given by Thomas et al. (2019) for local Seyfert 2 AGNs, and by Maiolino et al. (2008) for SF galaxies at different redshifts ranges (as labelled).

estimations and with estimations of the stellar mass of the host galaxies, mainly covering a wider range in masses, is needed to improve the $M-Z$ relation.

\section{CONCLUSION}

We compared the observational intensities of UV emission lines with results of photoionization models in order to obtain two semi-empirical calibrations between the metallicity of the NLR of type-2 active galactic nuclei and the $\mathrm{N} V \lambda 1240 / \mathrm{He}$ II $\lambda 1640$, $\mathrm{C} 43=\log [(\mathrm{C}$ IV $\lambda 1549+\mathrm{C}$ III] $\lambda 1909) / \mathrm{He}$ II $\lambda 1640]$, and C III] $\lambda 1909 / C$ IV $\lambda 1549$ emission-line ratios. We also derived a metallicity-free calibration between the ionization parameter and the C III] $\lambda 1909 / C$ IV $\lambda 1549$ emission-line ratio. We showed that the metallicity in NLRs of AGNs is lower by a factor of 2-3 than the metallicity in BLRs. Using the derived calibrations, we confirmed the recent result of the existence of a relation between the stellar mass of the host galaxy and its NLR metallicity. We were able to derive two $M-Z$ relations even though we consider that one of them is not statistically significant due to the small number of objects available to derive it. The adopted $M-Z$ relation was obtained using type-2 AGNs at $z$ between 1.6 and 3.8. Comparing our relation with the ones derived by Maiolino et al. (2008) for star-forming 
galaxies at high redshifts, we found that the $M-Z$ relation of type- 2 AGNs seems to complement the sequences towards higher masses following the same trend.

\section{ACKNOWLEDGEMENTS}

We are grateful to the anonymous referee for her/his very useful comments and suggestions that helped us to clarify and improve this work. OLD and ACK are grateful to FAPESP and CNPq. AFM is grateful to CAPES. MVC and GFH are grateful to CONICET. OLD is grateful to Dr. Sameshima and Dr. Miller for making their BLR metallicity estimations and $\alpha_{\mathrm{ox}}$ values available, respectively.

\section{REFERENCES}

Alende Prieto C., Lambert D. L., Asplund M., 2001, ApJ, 556, L63 Alende Prieto C., Lambert D. L., Asplund M., 2002, ApJ, 573, L137 Alloin D., 1973, A\&A, 27, 433

Baldwin J. A., Netzer H., 1978, ApJ, 226, 1

Batra N. D., Baldwin J. A., 2014, MNRAS, 439, 771

Berg D. A., Skillman E. D., Marble A. R., 2011, ApJ, 738, 2

Berg D. A., Skillman E. D., Henry R. C. B., Erb D. K., Carigi L., 2016, ApJ, 827,126

Blanc G. A., Kewley L., Vogt F. P. A., Dopita M. A., 2015, ApJ, 798, 99

Bornancini C. G., De Breuck C., de Vries W., Croft S., van Breugel W., Röttgering H., Minniti D., 2007, MNRAS, 378, 551

Bradley L. D., Kaiser M. E., Baan W. A., 2004, ApJ, 603, 463

Bresolin F., Kennicutt R. C., Garnett D. R., 1999, ApJ, 510, 104

Brown J. S., Martini P., Andrews B. H., 2016, MNRAS, 458, 1529

Castro C. S., Dors O. L., Cardaci M. V., Hägele G. F., 2017, MNRAS, 467, 1507

Contini M., 2017, MNRAS, 469, 3125

Davidson K., 1972, ApJ, 171, 213

Davidson K., 1977, ApJ, 218, 20

De Breuck C., Röttgering H., Miley G., van Breugel W., Best P., 2000, A\&A, 362,519

Dhanda N., Baldwin J. A., Bentz M. C., Osmer P. S., 2007, ApJ, 658, 804

Díaz A. I., Prieto M. A., Wamsteker W., 1988, A\&A, 195, 53

Dietrich M., Wilhelm-Erkens U., 2000, A\&A, 354, 17

Dietrich M., Hamann F., Shields J. C., Constantin A., Heidt J., Jäger K., Vestergaard M., Wagner S. J., 2003, ApJ, 589, 722

Dopita M. A. et al., 2006, ApJS, 167, 177

Dors O. L., Copetti M. V. F., 2005, A\&A, 437, 837

Dors O. L., Krabbe A. C., Hägele G. F., Pérez-Montero E., 2011, MNRAS, 415,3616

Dors O. L., Cardaci M. V., Hägele G. F., Krabbe Â. C., 2014, MNRAS, 443, 1291

Dors O. L., Cardaci M. V., Hägele G. F., Rodrigues I., Grebel E. K., Pilyugin L. S., Freitas-Lemes P., Krabbe Â. C., 2015, MNRAS, 453, 4102

Dors O. L., Arellano-Córdova K. Z., Cardaci M. V., Hägele G. F., 2017, MNRAS, 468, L113

Dors O. L., Agarwal B., Hägele G. F., Cardaci M. V., Rydberg C., Riffel R. A., Oliveira A. S., Krabbe A. C., 2018, MNRAS, 479, 2294

Edmunds M. G., Pagel B. E. J., 1984, MNRAS, 211, 507

Erb D. K., Steidel C. C., Shapley A. E., Pettini M., Reddy N. A., Adelberger K. L., 2006, ApJ, 646, 107

Esteban C., Peimbert M., Torres-Peimbert S., Rodríguez M., 2002, ApJ, 581,241

Esteban C., Bresolin F., Peimbert M., García-Rojas J., Peimbert A., MesaDelgado A., 2009, ApJ, 700, 654

Esteban C., García-Rojas J., Carigi L., Peimbert M, Bresolin F., LópezSánchez A. R., Mesa-Delgado A., 2014, MNRAS, 443, 624

Feltre A., Charlot S., Gutkin J., 2016, MNRAS, 456, 3354

Ferland G. J. et al., 2017, Rev. Mex. Astron. Astrofis., 53, 385

Ferland G. J., Baldwin J. A., Korista K. T., Hamman F., Carswell R. F., Phillips M., Wilkes B., Williams R. E., 1996, ApJ, 461, 683
García-Rojas J., Esteban C., 2007, ApJ, 670, 457

Gaskell C. M., Shields G. A., Wampler E. J., 1981, ApJ, 249, 443

Groves B. A., Heckman T. M., Kauffmann G., 2006, MNRAS, 371, 1559

Hamann F., Ferland G., 1992, ApJ, 391, L53

Hamann F., Ferland G., 1993, ApJ, 418, 11

Hamann F., Shields J. C., Cohen R. D., Junkkarinen V. T., Burbidge E. M., 1997, in Peterson B. M., Cheng F.-Z., Wilson A. S., eds, ASP Conf. Ser. Vol. 113, Emission Lines in Active Galaxies: New Methods and Techniques. Astron. Soc. Pac., San Francisco, p. 96

Hamann F., Korista K. T., Ferland G. J., Warner C., Baldwin J., 2002, ApJ, 564,592

Holweger H., 2001, AIPC, 598, 23

Kaspi S., Smith P. S., Netzer H., Maoz D., Jannuzi B. T., Giveon U., 2000, ApJ, 533, 631

Kennicutt R. C., Bresolin F., Garnett D. R., 2003, ApJ, 591, 801

Kewley L. J., Dopita M. A., 2002, ApJS, 142, 35

Kewley L. J., Ellison S. L., 2008, ApJ, 681, 1183

Kraemer S. B., Wu C.-C., Crenshaw D. M., Harrington J. P., 1994, ApJ, 435,171

Kumari N., Maiolino R., Belfiore F., Curti M., 2019, MNRAS, 485, 367

López-Sńchez. A., Esteban C., 2009, A\&A, 508, 615

López-Sńchez. A. R., Esteban C., Garcia-Rojas J., Peimbert M., Rodríguez M., 2007, ApJ, 656, 168

Ly C., Malkan M. A., Rigby J. R., Nagao T., 2016, ApJ, 828, 67

Maiolino R. et al., 2008, A\&A, 488, 463

Maiolino R., Mannucci F., 2018, A\&AR, 27, 3

Matsuoka K., Nagao T., Maiolino R., Marconi A., Taniguchi Y., 2009, A\&A, 503,721

Matsuoka K., Nagao T., Marconi A., Maiolino R., Mannucci F., Cresci G., Terao K., Ikeda H., 2018, A\&A, 616, L4

Mignoli M. et al., 2019, preprint (arXiv:190311085)

Miller B. P., Brandt W. N., Schneider D. P., Gibson R. R., Steffen A. T., Wu J., 2011, ApJ, 726, 20

Mollá M., Díaz A. I., 2008, MNRAS, 358, 521

Morisset C. et al., 2016, A\&A, 594, A37

Nagao T., Maiolino R., Marconi A., 2006, A\&A, 447, 863

Osmer P. S., 1980, ApJ, 237, 666

Osterbrock D. E., Ferland G., 2006, Astrophysics of Gaseous Nebulae and Active Galactic Nuclei, 2ndedn. Univ. Sci. Books, Mill Valley, CA

Pagel B. E. J., Edmunds M. G., Blackwell D. E., Chun M. S., Smith G., 1979, MNRAS, 189, 95

Peimbert M., 1968, ApJ, 154, 33

Pérez-Montero E., 2014, MNRAS, 441, 2663

Pérez-Montero E., Amorín R., 2017, MNRAS, 467, 1287

Pilyugin L. S., 2000, A\&A, 362, 325

Pilyugin L. S., 2001, A\&A, 369, 594

Pilyugin L. S., Grebel E. K., 2016, MNRAS, 457, 3678

Pilyugin L. S., Grebel E. K., Mattsson L., 2012, MNRAS, 424, 2316

Revalski M. et al., 2018, ApJ, 867, 88

Revalski M., Crenshaw D. M., Kraemer S. B., Fischer T. C., Schmitt H. R., Machuca C., 2018, ApJ, 856, 46

Sameshima H., Yoshii Y., Kawara K., 2017, ApJ, 834, 203

Sánchez S. F. et al., 2012, A\&A, 546, 2

Savaglio S. et al., 2005, ApJ, 635, 260

Shemmer O., Netzer H., 2002, ApJ, 567, L19

Shields G. A., 1974, ApJ, 191, 309

Shields G. A., 1976, ApJ, 204, 330

Shin J., Woo J.-H., Nagao T., Kim S. C., 2013, ApJ, 763, 58

Stasińska G., 2006, A\&A, 454, L127

Storchi-Bergmann T., 1991, MNRAS, 249, 404

Storchi-Bergmann T., Pastoriza M. Z., 1990, PASP, 102, 1359

Storchi-Bergmann T., Schmitt H. R., Calzetti D., Kinney A. L., 1998, AJ, 115,909

Suganuma M. et al., 2006, ApJ, 639, 46

Tananbaum H. et al., 1979, ApJ, 234, L9

Thomas A. D., Dopita M. A., Kewley L. J., Groves B. A., Sutherland R. S., Hopkins A. M., Blanc G. A., 2018, ApJ, 856, 89 
Thomas A. D., Kewley L. J., Dopita M. A., Groves B. A., Hopkins A. M., Sutherland R. S., 2019, ApJ, 874, 100

Uomoto A., 1984, ApJ, 284, 497

Viegas S. M., 2002, Rev. Mex. Astron. Astrofís., 12, 219

Yabe K. et al., 2014, MNRAS, 437, 3647

Yang C., Wang T., Ferland G. J., Dou L., Zhou H., Jiang N., Sheng Z., 2017, ApJ, 846, 150
York D. G. et al., 2000, AJ, 120, 1579

Zamorani G. et al., 1981, ApJ, 245, 357

Zhang Z. T., Liang Y. C., Hammer F., 2013, MNRAS, 430, 2605

This paper has been typeset from a $\mathrm{TE}_{\mathrm{E}} \mathrm{X} / \mathrm{L} \mathrm{T} \mathrm{E} \mathrm{X}$ file prepared by the author. 